Linköping Studies in Science and Technology

Dissertation No. 2051

\title{
Learning to Make Safe Real-Time Decisions Under Uncertainty for Autonomous Robots
}

\section{Olov Andersson}
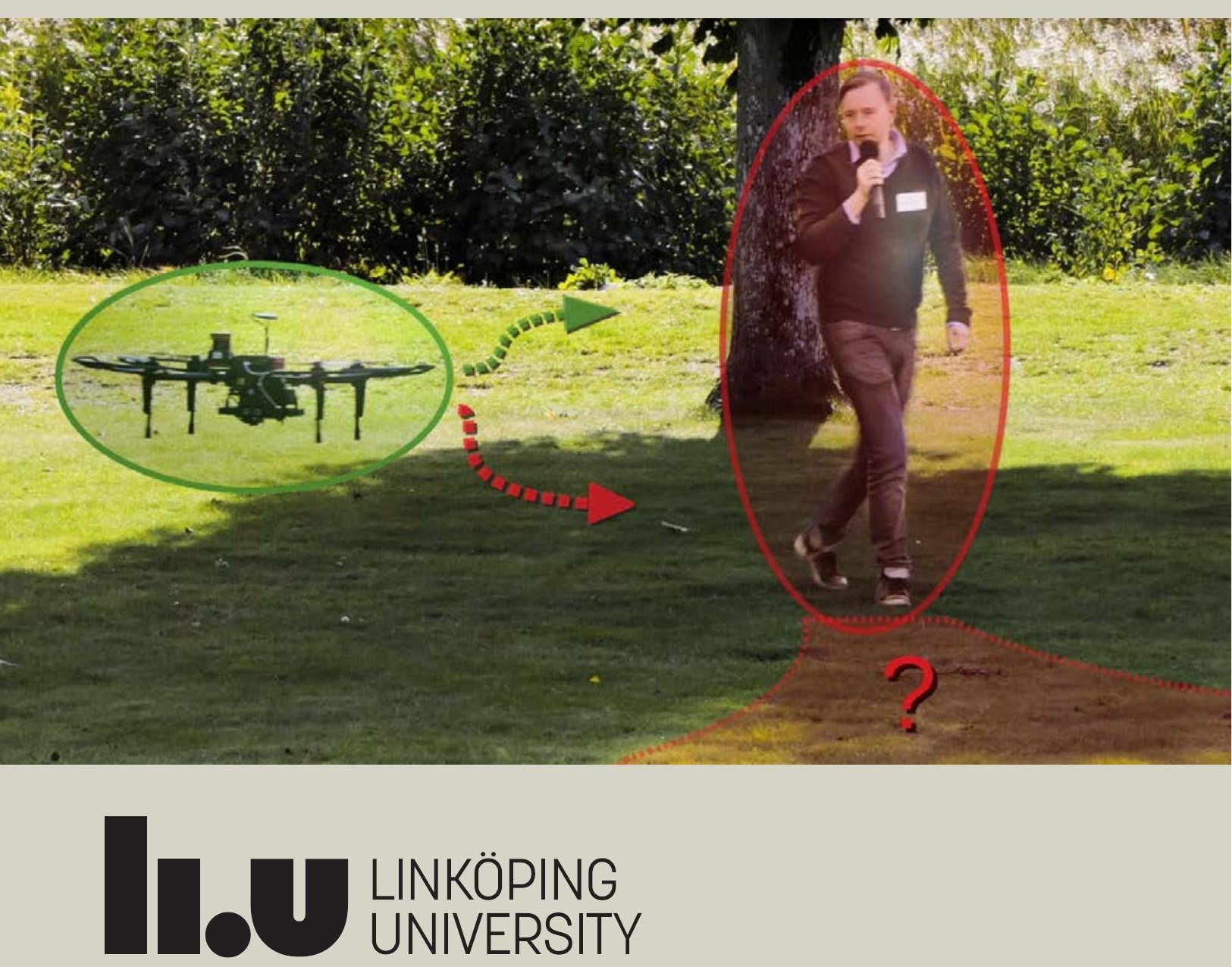


\title{
Learning to Make Safe Real-Time Decisions Under Uncertainty for Autonomous Robots
}

\author{
Olov Andersson
}

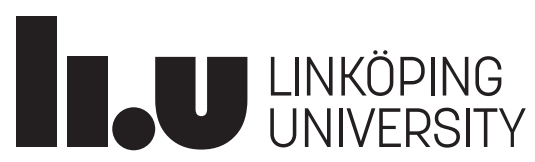

Linköping University

Department of Computer and Information Science Artificial Intelligence and Integrated Computer Systems SE-581 83 Linköping, Sweden 


\section{Edition 1:1}

(C) Olov Andersson, 2020

The cover shows an autonomous quadcopter learning to safely avoid the author of this thesis during a WASP research demonstration at Gränsö Slott, September 18, 2019. Photo by Thor Balkhed, Linköping University. Included papers reprinted with permission.

ISBN 978-91-7929-889-0

ISSN $0345-7524$

URL http://urn.kb.se/resolve?urn=urn:nbn:se:liu:diva-163419

Published articles have been reprinted with permission from the respective copyright holder.

Typeset using $\mathrm{ET}_{\mathrm{E}} \mathrm{X}$

Printed by LiU-Tryck, Linköping 2020 


\section{POPULÄRVETENSKAPLIG SAMMANFATTNING}

Denna avhandling undersöker hur metoder från artificiell intelligens och maskininlärning kan användas för att förbättra robotars förmåga att själva fatta säkra och effektiva beslut när de ska navigera ute i världen. Robotar har hittils främst haft smala tillämpningar inom industrin, men väntas nu allt mer gå bortom sådana kontrollerade miljöer till att kunna agera med en hög grad av autonomi på allmänna platser, såsom i trafiken eller på arbetsplatser. Med autonomi avses att roboten själv förväntas kunna fatta beslut utan mänsklig hjälp. Sådant beslutsfattande löses ofta rent matematiskt genom optimeringsbaserade styr- och planeringsmetoder. Till skillnad från industrimiljöer är världen utanför däremot tyvärr ofta både svårtolkad och svårförutsägbar. Autonoma robotar måste därför även ta hänsyn till den osäkerhet som kan uppstå i farliga situationer. Sådan osäkerhet kommer från flera olika källor. En del kommer från robotens imperfekta interna modeller av verkligheten, men osäkerhet kan också vara en sidoeffekt av begränsningar i vad en robot kan förnimma, så kallad partiell observerbarhet från sensorbegränsningar och ocklusion.

Oavsett källan så blir det slutliga beslutsproblemet ofta tyvärr ohanterligt svårlösligt när man rent matematiskt tar hänsyn till all osäkerhet. Detta innebär en stor utmaning eftersom verkligheten också är dynamisk, den kommer inte att stanna för att vänta på att roboten ska fatta ett beslut. Autonoma robotar som navigerar på offentliga platser, till exempel i trafik, måste kunna ta beslut i realtid. Osäkerhet är därför i praktiken ofta matematiskt försummad när robotar löser styr- och beslutsproblem, med potentiellt katastrofala sidoeffekter som följd när något oväntat händer.

Målet med denna avhandling är att nyttja nya framsteg inom området maskininlärning för att robotar även under osäkerhet ska kunna fatta både effektiva och säkra beslut i realtid. Vi undersöker flertalet olika metoder, från probabilistisk- till djupinlärning, och kombinerar även dessa med de klassiska optimeringsbaserade styr- och planeringmetoder som redan idag tillämpas inom robotik. Vi använder ett tillämpningsdrivet tillvägagångssätt, förankrat i navigeringsexperiment med verkliga autonoma drönare, för att angripa flera olika delar av detta problem. Från att reducera osäkerheten i problemet genom att lära sig bättre modeller av verkligheten, till att direkt lära sig att approximera beslutsfattandet, samtidigt som man försöker säkerställa de säkerhets- och realtidskrav som krävs för autonomi i en dynamisk och osäker verklighet. 


\begin{abstract}
Robots are increasingly expected to go beyond controlled environments in laboratories and factories, to act autonomously in real-world workplaces and public spaces. Autonomous robots navigating the real world have to contend with a great deal of uncertainty, which poses additional challenges. Uncertainty in the real world accrues from several sources. Some of it may originate from imperfect internal models of reality. Other uncertainty is inherent, a direct side effect of partial observability induced by sensor limitations and occlusions. Regardless of the source, the resulting decision problem is unfortunately computationally intractable under uncertainty. This poses a great challenge as the real world is also dynamic. It will not pause while the robot computes a solution. Autonomous robots navigating among people, for example in traffic, need to be able to make split-second decisions. Uncertainty is therefore often neglected in practice, with potentially catastrophic consequences when something unexpected happens. The aim of this thesis is to leverage recent advances in machine learning to compute safe real-time approximations to decision-making under uncertainty for real-world robots. We explore a range of methods, from probabilistic to deep learning, as well as different combinations with optimization-based methods from robotics, planning and control. Driven by applications in robot navigation, and grounded in experiments with real autonomous quadcopters, we address several parts of this problem. From reducing uncertainty by learning better models, to directly approximating the decision problem itself, all the while attempting to satisfy both the safety and real-time requirements of real-world autonomy.
\end{abstract}

This work has been supported by the Wallenberg Al, Autonomous Systems and Software Program, the Swedish Foundation for Strategic Research (SSF) project Symbicloud and the ELLIIT Excellence Center at Linköping-Lund for Information Technology, in addition to those sources already acknowledged in the individual papers. 


\section{Acknowledgements}

I am grateful for the support of my primary advisor Patrick Doherty, giving me the time and resources to explore interesting research directions. I am also grateful for the fruitful discussions on Bayesian learning with my co-advisor Mattias Villani. To all my colleagues in the AlICS division, a big thanks for the positive work environment. Special thanks to the UASTech lab in particular. Mariusz, Piotr, Cyrille, Tommy and Karol, you have been a fount of knowledge on UAVs and field robotics. I am also grateful for the support from friends and family, including all my relatives up north. Thank you for putting up with me during these intense years. Special thanks also to my late friend Thomas for our stimulating discussions. Finally, I want to thank my parents Eva and Henning, this would not have been possible without you.

Linköping, 13 March 2020

Olov Andersson 



\section{Contents}

Abstract iii

Acknowledgments $\quad$ v

Contents vii

List of Figures $\quad$ xi

List of Tables $\quad$ xv

1 Introduction 1

$1.1 \quad$ Uncertainty in the Real World - A Motivating Example . . . . . . . . . . . . . . 2

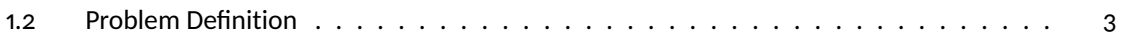

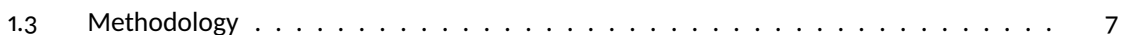

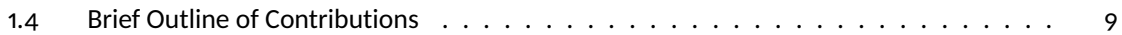

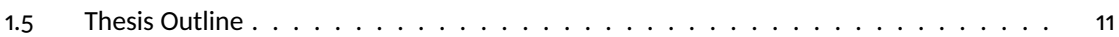

\begin{tabular}{|ll|}
\hline 2 & Learning and Inference
\end{tabular}

$2.1 \quad$ Preliminaries . . . . . . . . . . . . . . . . . . . . . . . 13

$2.2 \quad$ Learning and Parameteric Models $\ldots \ldots \ldots \ldots$. . . . . . . . . . . . . . 14

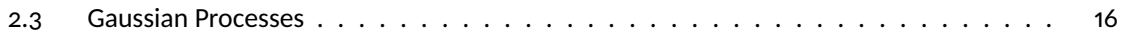

$2.4 \quad$ Deep Learning . . . . . . . . . . . . . . . . . . . . . . . . . . . . . . . . 18

3 Decision, Planning and Control under Uncertainty 21

$3.1 \quad$ Making Safe Decisions Under Uncertainty . . . . . . . . . . . . . . . . . . . 21

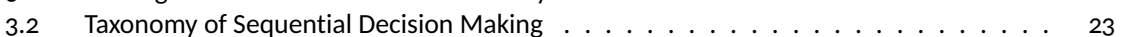

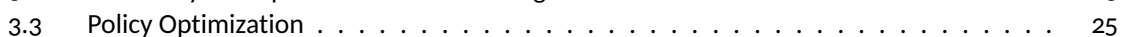

3.4 Safe Real-Time Planning Under Uncertainty . . . . . . . . . . . . . . . . . 30

\begin{tabular}{|ll|l}
4 & Summary and Discussion & 39
\end{tabular}

$4.1 \quad$ Summary of Contributions . . . . . . . . . . . . . . . . . . . . 39

4.2 Discussion and Future Work $\ldots \ldots \ldots \ldots \ldots$. . . . . . . . . . . 46

\begin{tabular}{ll}
\hline Bibliography & 49
\end{tabular}

5 Paper I 59

5.1 Introduction . . . . . . . . . . . . . . . . . . . . 59

5.2 Stochastic Trajectory Optimization . . . . . . . . . . . . . . . . . 61

5.3 Human Obstacle Models $\ldots \ldots \ldots \ldots$. . . . . . . . . . . . . . . . 63

5.4 Trajectory-Policy Approximations . . . . . . . . . . . . . . . . . . . . . 65

5.5 Case Study: Safe Quadcopter MPC . . . . . . . . . . . . . . . . . . . . . . 68 


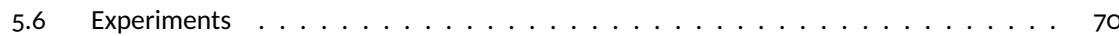

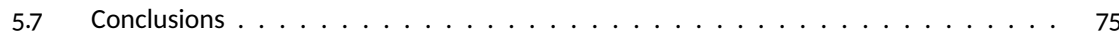

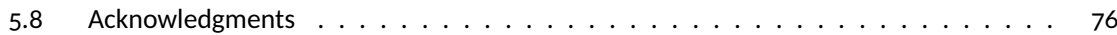

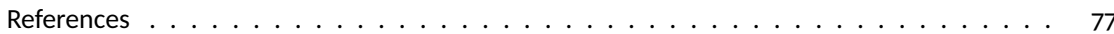

\begin{tabular}{lll}
\hline & Paper II & 83 \\
\hline
\end{tabular}

6.1 Introduction ....................... 83

6.2 Problem formulation . . . . . . . . . . . . . . . . . . . . . . . . 87

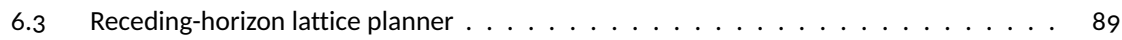

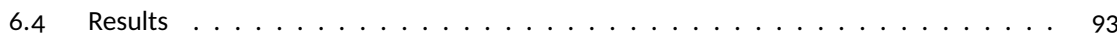

6.5 Conclusions and Future Work . . . . . . . . . . . . . . . . . . . . . . 101

$6.6 \quad$ Acknowledgments . . . . . . . . . . . . . . . . . . . . . . . 101

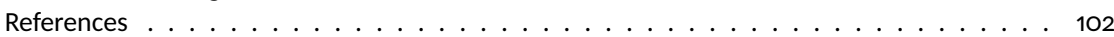

\begin{tabular}{lll}
\hline 7 & Paper III & 107
\end{tabular}

$7.1 \quad$ Safe Motion Planning in Dynamic Uncertain Environments . . . . . . . . . . . . . . . 110

$7.2 \quad$ Uncertainty from Dynamic Obstacles such as Pedestrians and Vehicles . . . . . . . . . 114

$7.3 \quad$ Learning Risk-Adjusted Trajectory Approximations . . . . . . . . . . . . . . . . . 118

7.4 Case Study I: Safe MPC Obstacle Avoidance under Motion Uncertainty. . . . . . . . . 127

7.5 Case Study II: Safe Motion Planning under Limited Visibility in DUE. . . . . . . . . . . 135

7.6 Limitations and Future Work . . . . . . . . . . . . . . . . . . . . . . 142

7.7 Conclusions . . . . . . . . . . . . . . . . . . . . . . . . . . . . . . 143

$7.8 \quad$ Acknowledgments . . . . . . . . . . . . . . . . . . . . . . . . . . . . 144

References ............................... 144

\begin{tabular}{lll}
\hline & Paper IV & 151
\end{tabular}

$8.1 \quad$ Introduction $\ldots \ldots \ldots \ldots \ldots \ldots \ldots \ldots \ldots \ldots$

$8.2 \quad$ Trajectory Optimization. . . . . . . . . . . . . . . . . . . . . . . . . . . . 153

8.3 Learning Deep Policy Approximations . . . . . . . . . . . . . . . . . . . . . . . . . . 154

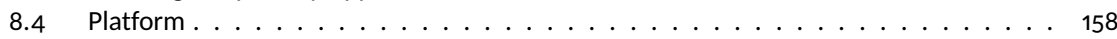

$8.5 \quad$ Experiments $\ldots \ldots \ldots \ldots \ldots \ldots \ldots \ldots \ldots$

8.6 Conclusions . . . . . . . . . . . . . . . . . . . . . . . . . . 164

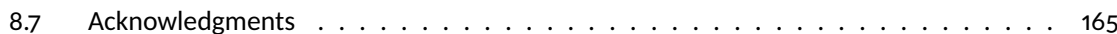

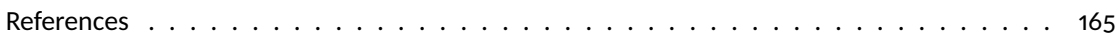

9 \begin{tabular}{ll}
\hline Paper V & 171
\end{tabular}

$9.1 \quad$ Introduction $\ldots \ldots \ldots \ldots \ldots \ldots \ldots \ldots$

$9.2 \quad$ Toy Example: Robot Obstacle Avoidance . . . . . . . . . . . . . . . . . . . . . . 173

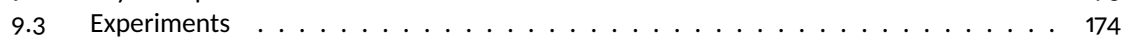

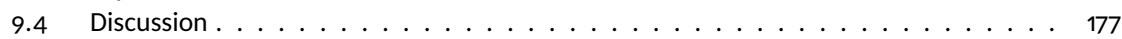

$9.5 \quad$ Acknowledgments . . . . . . . . . . . . . . . . . . . . . 178

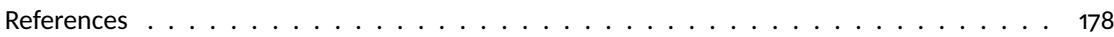

10 Paper Vl 183

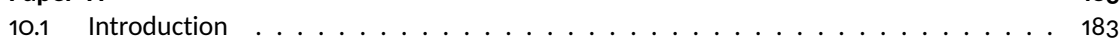

10.2 Problem Definition . . . . . . . . . . . . . . . . . . . . . . . . . . 185

10.3 Learning the Dynamics . . . . . . . . . . . . . . . . . . . . . . . . . 186

10.4 Constrained Trajectory Optimization . . . . . . . . . . . . . . . . . . . . . . . . . 189

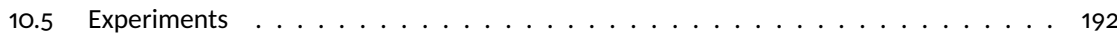

10.6 Conclusions $\ldots \ldots \ldots \ldots \ldots \ldots \ldots \ldots$

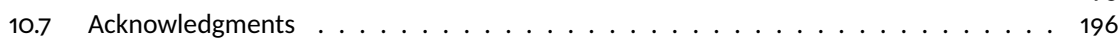

References . . . . . . . . . . . . . . . . . . . . . 196

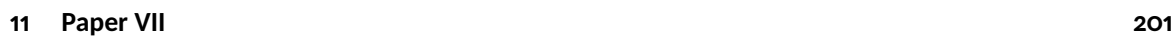

11.1 Introduction . . . . . . . . . . . . . . . . . . . . 201 
$11.2 \quad$ Spatial Point Process for Disaster Response $\ldots \ldots \ldots$. . . . . . . . . . . . . 203

$11.3 \quad$ Online Learning Using INLA . . . . . . . . . . . . . . . . . . . . . . . . . . . . 206

11.4 Planning Exploratory Moves . . . . . . . . . . . . . . . . . . . . . . . . . . . 209

11.5 Experiments . . . . . . . . . . . . . . . . . . . . . . . 212

11.6 Conclusions . . . . . . . . . . . . . . . . . . . . . . . . . . . 215

References . . . . . . . . . . . . . . . . . . . . . . . . . 217 



\section{List of Figures}

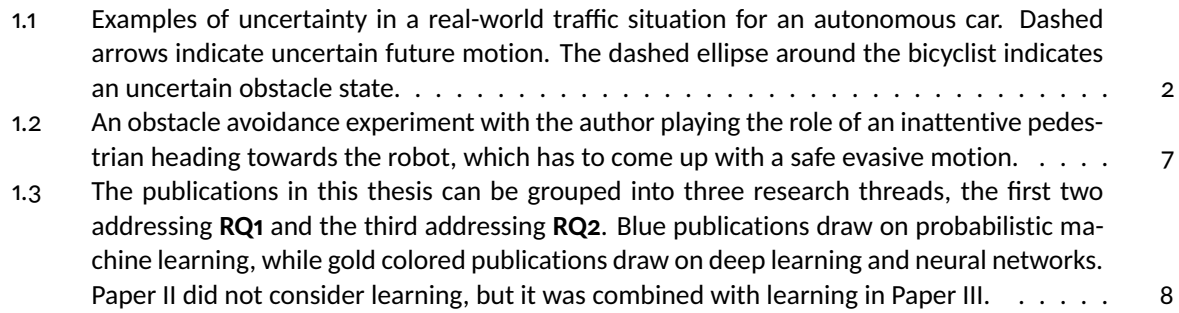

2.1 Sparse approximation to a $f: \mathbb{R}^{1} \rightarrow \mathbb{R}^{1}$ Gaussian process inferred from data points in black. Predicted mean and $95 \%$ probability intervals in blue and cyan respectively. The red crosses represent the inducing points of the sparse approximation. . . . . . . . . . 18

2.2 Architecture of a fully-connected neural network with two hidden layers for learning some function $f: \mathbb{R}^{3} \rightarrow \mathbb{R}^{2}$. Artificial neurons are shown in white and their inputs are represented by arrows. $\ldots \ldots \ldots \ldots \ldots$

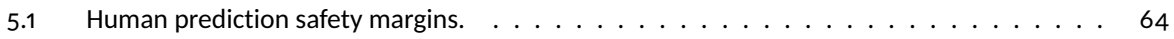

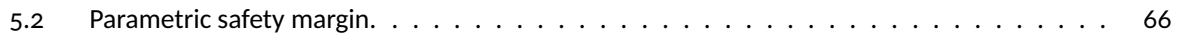

$5.3 \quad$ The LinkQuad Quadcopter. . . . . . . . . . . . . . . . . . . . . . . . . . 70

5.4 The intersection scenario, crossing a street. . . . . . . . . . . . . . . . 71

5.5 Bayesian policy optimization of safety parameters for the intersection scenario. . . . . . . 72

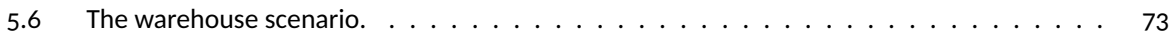

$5.7 \quad$ Bayesian policy optimization of safety parameters for the warehouse scenario. . . . . . . . 74

5.8 Quadcopter avoiding one human obstacle. . . . . . . . . . . . . . . . 76

5.9 Quadcopter avoiding two human obstacles. . . . . . . . . . . . . . . . . . 76

6.1 Example indoor 3D warehouse scenario with both static and dynamic obstacles, including humans (red) on the ground, and other quadcopters (orange) flying at varying altitudes. The faded spheres are predictions of future motion. $\ldots \ldots \ldots . \ldots . \ldots 4$

6.2 Examples of a bad (top) and a good (bottom) solution to the receding-horizon motion planning problem for a quadcopter that is operating in an indoor environment. The top figure is when relevant terminal cost (cost-to-go) is underestimated, and the bottom figure when the terminal cost is calculated in a systematic way. . . . . . . . . . . . . . . . . 89

6.3 Motion primitives for a quadcopter from where the vehicle is hovering to different neighboring states on the high resolution lattice grid that is used during the temporal planning

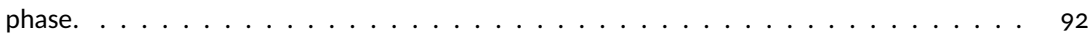

6.4 Illustration of the motion planning and control architecture that is used for the quadcopter platform. The modules that are colored in blue are considered in this work. . . . . . . . . 95 
6.5 High resolution motion primitives from $\mathbf{s}_{i}=\left(0, \mathbf{v}_{d, i}(0), 0\right)$ to different states on the lattice grid. The blue, red and black trajectories are from $\mathbf{v}_{d, i}(0)=(0,-2,0), \mathbf{v}_{d, i}(0)=(0,0,1)$

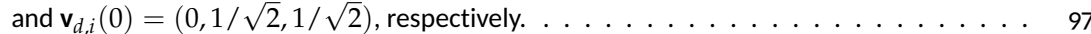

6.6 This figure shows a scenario where the UAV has to find a safe spot to move to, and wait at, until an approaching obstacle has passed by. Such situations necessitates a temporal lattice planner with a possible stand-still or wait action. . . . . . . . . . . . .

6.7 The number of state evaluations required to plan around an obstacle (wall of increasing length) using different approaches. A baseline lattice grid without time (Yellow), a grid with wait time (Blue) and a grid with time (Red). Right: Proportion between the two time-based variants against the baseline. . . . . . . . . . . . . . . . 100

7.1 Example of a motion planning in a dynamic uncertain environment, e.g. a typical populated office or warehouse. There is uncertainty in the motion of obstacles such as people, which is compounded by occlusions in the environment. To safely navigate this environment the robot needs to take into account risks induced by uncertainty from obstacle motion and occlusion. A classical minimum-time plan to the destination can result in collisions from unexpected obstacle motion, such as the yellow arrow from the occluded obstacle above. 108

7.2 Various safety constraint approximations applied to a pedestrian predictive distribution at

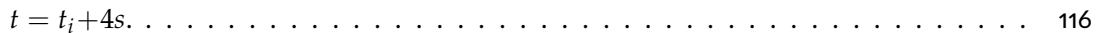

7.3 Non-parametric estimate of actual safety probability for each position at $t=t_{i}+4 \mathrm{~s}$ from 50000 motion samples. $\ldots \ldots \ldots \ldots \ldots \ldots$

7.4 The stochastic motion planning problem is non-convex and non-conservative solutions require a non-linear feed-back policy to evade only if future obstacle motion is unsafe. $\quad$. . . 119

7.5 Simple example showing the spherical support (gray) of a parameterized risk function, reflecting the risk induced by proximity to the uncertain expected future obstacle positions at

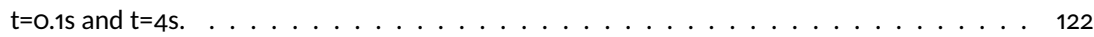

7.6 Left: The quadcopter used in the experiments and an inattentive pedestrian walking towards it. Right: Top-down symbolic view of the pedestrian (red) and its mean prediction (yellow), as well as the quadcopter (green) and its planned trajectory to goal (cross). . . . . 129

7.7 The intersection scenario, crossing pedestrian traffic. Robot in green, obstacles in red and their mean prediction in yellow. . . . . . . . . . . . . . . . . . . 131

$7.8 \quad$ Bayesian policy optimization of safety parameters for the intersection scenario. . . . . . . 131

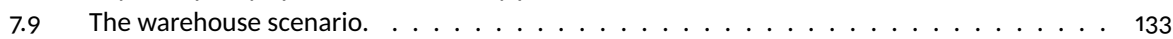

$7.10 \quad$ Bayesian policy optimization of safety parameters for the warehouse scenario. . . . . . . . 134

7.11 Quadcopter avoiding one human obstacle. . . . . . . . . . . . . . . . 136

7.12 Quadcopter avoiding two human obstacles. . . . . . . . . . . . . . . . . . 136

7.13 Example indoor 3D warehouse scenario with both static and dynamic obstacles, including humans (red) on the ground, and other quadcopters (orange) flying at varying altitudes. The faded spheres are predictions of future motion. . . . . . . . . . . . . . . 138

7.14 The lattice planner connects states in a regular lattice via motion primitives (exemplified above) generated by numerical optimal control. . . . . . . . . . . . . . . 138

7.15 Blind corner example with a visibility frontier in red. Conventional time-optimal trajectory planning tends to hug corners (c.f. Fig. 7.1), while safety under uncertainty from occluded dynamic obstacles may require prioritizing visibility. . . . . . . . . . . . . . . 139

7.16 Learned task (left) and safety constraint (right) surfaces for risk parameters in the blind corner scenario. Top row shows $\theta_{v}$ against $\theta_{p}$, while the bottom row shows $\theta_{v}$ against $\theta_{\text {limit }} \cdot{ }_{1} \quad 142$

7.17 The learned risk adjustment generalizes to occlusions from passing through doors. Here it has no option but to go through even though its visibility is poor. Instead it slows down on the threshold, correctly reflecting that going fast through doors carries a risk of running into unobserved obstacles passing by. . . . . . . . . . . . . . . . . . 143

8.1 Training procedure for deep neural network policy approximations. . . . . . . . . . . . . 155

8.2 The Bitcraze Crazyflie 2.0 nano-quadcopter. . . . . . . . . . . . . . . . . . . . . 158

8.3 Safety margin for stochastic collision avoidance. . . . . . . . . . . . . . . . . . . . . . . 159

8.4 The warehouse scenario with three obstacles. . . . . . . . . . . . . . . . . . . . 160 
$8.5 \quad$ Computational cost of action selection. . . . . . . . . . . . . . . . . . . 162

8.6 On-board DNN quadcopter experiments. Top shows it dodging one human obstacle. Bottom shows it flying a rectangular pattern while avoiding one human obstacle. . . . . . . . . . 164

9.1 Left: Simplified RL environment with moving obstacles (velocities represented as arrows). Right: Collision example, the agent rams into the top obstacle. . . . . . . . . . . . . 173

9.2 Reward curves for learning with a) Static initial state and obstacles (o.5M steps) (b) Random initial state and moving obstacles (10M steps) (c) Log-plot out to 40M steps. Runs are either

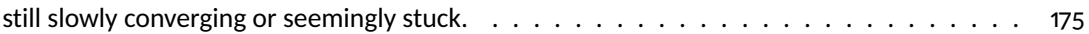

9.3 Exploration noise $\sigma_{\pi}$ vs. convergence rate for a) deterministic environment, b) stochastic environment. c) Experiment testing distribution over local minima. . . . . . . . . . . . . . 177

10.1 Forward simulation of planned actions for a quadcopter control agent told to reach position $x=1$. While it plans in a 10-dimensional state-action space, only the target variable is shown. 191

10.2 The success rate per episode in terms of the percentage that completed without violating pole or track constraints, averaged over 50 runs. . . . . . . . . . . . . . . 193

10.3 Log plot of the final cart distance to the target position while keeping the pole balanced. The results are averaged over 50 runs. $\ldots \ldots \ldots \ldots 3$

10.4 Positional control of quadcopter commanded to fly a rectangle pattern. Blue indicates acceleration, and red deceleration, by getting the quadcopter to tilt in or against the direction of movement. Green is constant velocity. . . . . . . . . . . . . . . . . . . . . 194

10.5 Quadcopter $x, y$ position and velocity while flying the rectangle pattern. The dotted line represents the indoor domain constraint on velocity. $\ldots \ldots \ldots$. . . . . . . . . . 195

11.1 Top: Map of the town of Gamleby, Sweden with buildings (orange), forest (dark green), fields (light green), roads (light grey) and water (dark grey) color coded. Bottom: a sample realization from the model showing the population intensity overlaid by persons in the area (filled dots), detected persons (green circles) and injured persons (blue crosses). . . . . . . 204

11.2 Search scenario using MCT S Jump, overlaid on heatmap for expected number of detectable

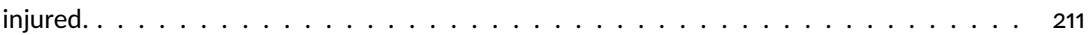

11.3 Comparing the proportion of injured found as a function of search time (minutes) for the different strategies (lawnmower, MCTS, and MCTS Jump). The rows correspond to each of the four scenarios A to D (top down). The graphs show the mean proportion of injured found as a function of search time (solid line) for the three strategies over 30 replicates, as well as the $95 \%$ confidence bands for the mean (darker regions) and 95\% predictive bands for individual proportions in individual replicated datasets (lighter regions). The final column shows that same properties, but for the differences in proportions between MCTS and MCTSjump. .......................... 217

11.4 True and inferred population and injury maps for scenario 6. All intensity plots are based on detectable persons only. The first row displays the ground truth used for simulating the data, showing the expected number of persons per cell, the probability of being injured in each cell, and the expected number of injured in each cell. The second row shows $\exp \left(E\left(\xi_{q}\right)\right), E(q)$, and $E(\lambda r q)$, with expectations with respect to the posterior after 14 search iterations. The third row is equivalent to the second row, but after 91 iterations of

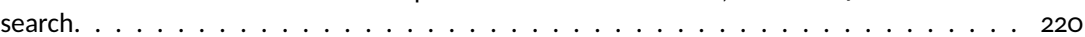

11.5 Sensitivity analysis for Scenario B. The first row displays the results for the prior used in the paper and is the same as the second row of Figure 11.3 The second row changes the prior mean for $\beta$ on buildings and roads in the population intensity to be two standard deviations larger than the baseline prior. The third row changes the prior mean for $\beta$ on buildings in the injury probability to be two standard deviations larger than the baseline prior. . . . . . 221 



\section{List of Tables}

5.1 Results from intersection scenario for different algorithms and target safety levels. Actual safety level estimated over $12 \mathrm{~h} . \quad \ldots \ldots \ldots \ldots \ldots$. . . . . . . . . . . . .

5.2 Warehouse scenario for different target safety levels. Actual safety level estimated over $12 \mathrm{~h} .6$

5.3 Optimization results from warehouse scenario with different numbers of humans . . . . . 75

6.1 Results from 100min of the difficult indoor warehouse scenario with moving noncooperative obstacles in 3D. Ablation study of proposed Receding Horizon Motion Planner (RHLP) against three restricted baselines. Time to goal and Nodes evaluated are averages per randomized goal, and plan, respectively. . . . . . . . . . . . . . . . . 101

7.1 Results from intersection scenario for different algorithms and target safety levels. Actual safety level estimated over $12 \mathrm{~h} . \quad \ldots \ldots \ldots \ldots \ldots \ldots$

7.2 Warehouse scenario for different target safety levels. Actual safety level estimated over $12 \mathrm{~h}$. 133

7.3 Optimization results from warehouse scenario with different numbers of humans . . . . . 135

7.4 Blind corner scenario for different target safety levels. Actual safety level estimated over $24 \mathrm{~h}$. 141

$8.1 \quad$ One non-cooperative moving obstacle. . . . . . . . . . . . . . . . . . 161

8.2 Three non-cooperative moving obstacles. . . . . . . . . . . . . . . . . . . . . 162

11.1 Scenario Settings. Covariates and spatial fields in data generating process and inferred model.

$B=$ buildings, $R=$ roads, $W=$ water, $F=$ forest, $G_{i}=$ Gaussian no $i, S=$ spatial field. Deviations

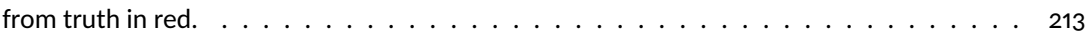

11.2 Time until half of injured have been found (minutes) . . . . . . . . . . . . . . . . . . . 214 

CHAPTER

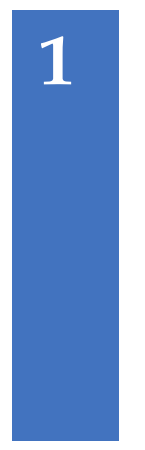

\section{Introduction}

Over the next few decades, robotic automation is projected to reach beyond factories and laboratories, to increasingly enter real-world public spaces and homes. Several forecasts (Arntz and Zierahn, 2016. Frey and Osborne, 2017) predict that society is on the verge of a wider artificial intelligence (AI) driven wave of automation with wide-spread economic impact. While traditional robots in manufacturing can usually be manually programmed for a small number of scenarios, the real world is both complex and uncertain. Manually programming how a robot should behave in every possible situation that can arise, for example in traffic, busy workplaces or cluttered homes, is a daunting task. Such robots require a high level of autonomy, the capacity to make decisions on their own. However, poor decisions in the real world can cause damage to property or even loss of life. This imposes stringent requirements on safety and robustness for such autonomous robots acting in the real world. Further, since the real world is dynamic, it has to arrive at safe decisions within the time constraints set by its environment, for example split-second decisions to take evasive action in traffic.

However, real-world robots have limited computational capacity, while decision making under uncertainty is computationally a hard problem. Uncertainty in real-world situations is therefore often neglected to allow real-time performance. As is becoming increasingly clear, this can have potentially undesirable ramifications for safety. In this thesis we instead seek tractable real-time approximations to decision making under uncertainty that can still satisfy desired safety requirements. Guided by robotics applications, we address different types of uncertainty in the decision problem. We build on the strengths of both recent machine learning approaches, as well as conventional optimization-based planning from robotics and control, to find such safe and effective real-time approximations for real-world robots. 


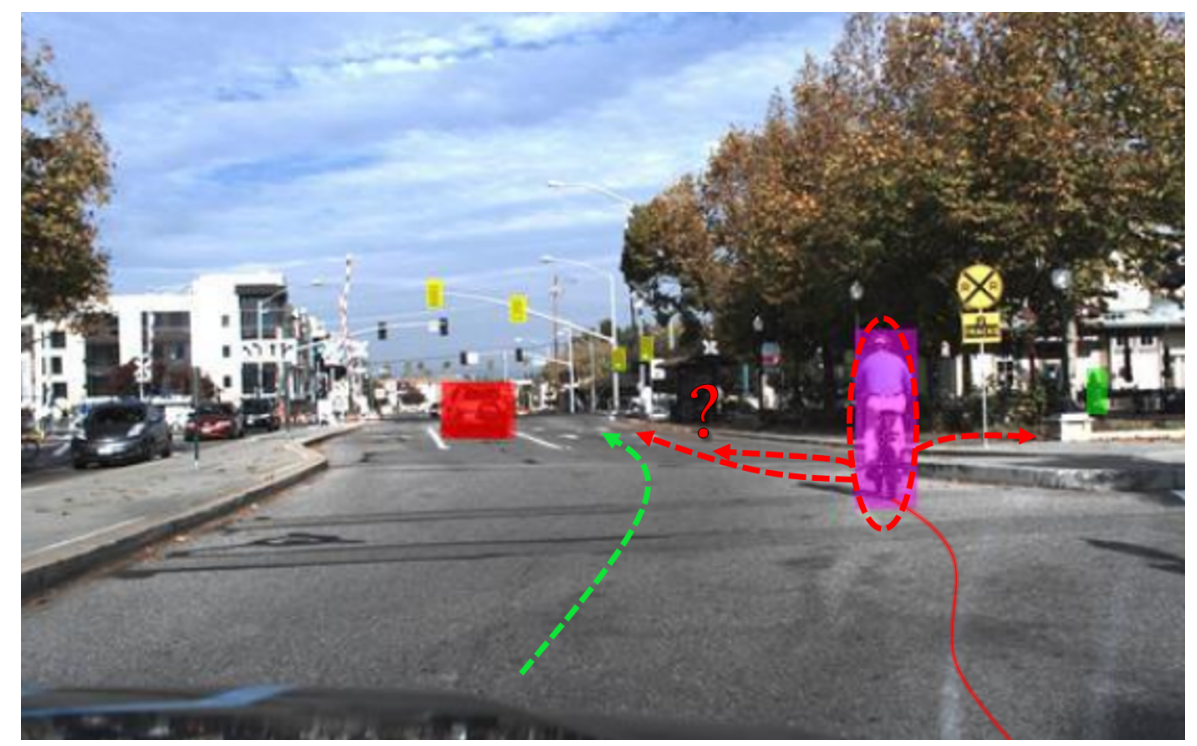

Figure 1.1: Examples of uncertainty in a real-world traffic situation for an autonomous car. Dashed arrows indicate uncertain future motion. The dashed ellipse around the bicyclist indicates an uncertain obstacle state.

\subsection{Uncertainty in the Real World - A Motivating Example}

While autonomous robots already come in many shapes, ranging from simple cleaning and lawnmower robots, to drones and biologically inspired quadrupeds such as Spot (Boston Dynamics, 2019), the type of autonomous robot currently getting the most attention is likely self-driving cars. By adding autonomy to cars, they become robots made to transport people. These are more than big enough to cause real injury, making safety crucial. They also have to be autonomous in potentially open-ended environments with many sources of uncertainty, including the interaction with other traffic participants.

Here we use autonomous vehicles as an example to illustrate some common sources of uncertainty that autonomous robots have to contend with in the real world. A typical traffic situation from the Udacity autonomous driving data set (Udacity, 2016) is shown in Figure 1.1 .

Broadly speaking, we can divide this uncertainty into pertaining to either the current state of the world, or how this state will change in the future:

(i) Current state estimates of the world will be uncertain because sensors such as cameras, radar and lidar are imperfect. This includes the position, velocity and type of obstacles, but also the state of the robot itself. 
(ii) Future state predictions are inherently uncertain because a robot's internal models of the world will never be perfect. The future motion of pedestrians, bicyclists and other vehicles can only be guessed at, and the uncertainty increases the farther it tries to predict. However, there is also uncertainty in the ego-motion of the robot itself. Even though it may know where it plans to go, imperfect control and environmental disturbances such as wheel slippage or gusts of wind can introduce uncertainty.

An example of what can happen when an autonomous robot makes poor decisions is exemplified by a recent tragic accident involving an Uber autonomous test vehicle, where it hit a jaywalking pedestrian pushing a bike under poor lighting conditions. An official investigation (NTSB, 2019) found several problems leading up to the crash, including that the system had simply not been designed with jaywalking pedestrians in mind. Over the course of five seconds, from initial detection to impact, the system failed to slow down because it oscillated between classifying the pedestrian as various static and dynamic obstacles. Each time acting on the new classification as if it was certain without regard to its history. We can only speculate about what could have changed the outcome of this tragic accident, but an uncertainty-aware robot can react to the mere possibility of a pedestrian by slowing down.

While this is an area attracting increasing interest from the research community, how to tractably incorporate uncertainty into real-time decisionmaking for autonomous robots still remains an open problem. As autonomous robots increasingly enter public spaces such as streets and workplaces, society is in need of effective solutions. This thesis aims to contribute to the growing body of knowledge on this topic by leveraging recent techniques from machine learning, where we focus on finding approximations that satisfy the computational and safety needs of autonomous robots. Grounded in real-world experiments, we explore several methods to address sources of uncertainty arising in robotics applications.

\subsection{Problem Definition}

The robot has to make decisions under uncertainty, which is usually formalized (Bertsekas, 2011) as finding some action $\boldsymbol{u}$ minimizing the expected cost over uncertain problem state variables $x$,

$$
\underset{\boldsymbol{u}}{\arg \min } \mathbb{E}_{\boldsymbol{x}}[c(\boldsymbol{x}, \boldsymbol{u})] .
$$

The cost function $c(\boldsymbol{x}, \boldsymbol{u})$ is selected to reflect problem desiderata, which can range from the time it takes to reach a goal destination or the cost of fuel used, to any harm imposed on its environment. Here $x$ is a vector of 
uncertain state variables, for example due to imperfect sensors in (i) above. This may include the position of the obstacles in relation to the robot, their velocity, or type of obstacle such as the incident in the previous example. However, the expectation operator $\mathbb{E}[$.$] now forces the robot to make deci-$ sions with regard to the cost of all possible outcomes of this uncertain state $x$, weighted by the probability of each outcome. The above decision rule is also famously the definition of a rational agent, a common model used to study human decision-making in economics, and later adopted by artificial intelligence

However, many problems exhibit sequential dependence between earlier decisions and the state variables $x$. For example, to reach a destination the position of an autonomous car can only be indirectly manipulated through a sequence of steering, acceleration and braking actions. As a robot interacts with the world, each action $\boldsymbol{u}_{t}$ taken at time $t$ will change the next world state $\boldsymbol{x}_{t}$ according to some uncertain motion model $p\left(\boldsymbol{x}_{t+1} \mid \boldsymbol{x}_{t}, \boldsymbol{u}_{t}\right)$. This uncertainty is of type (ii) above, resulting from imperfect models, both of its own future motion and that of other agents.

The agent now needs to plan a sequence of actions to fulfill its objective. It therefore has to solve a harder sequential decision problem,

$$
\underset{\pi(x)}{\arg \min } \mathbb{E}_{\boldsymbol{x}_{t: t+H}}\left[c\left(\tau_{t: t+H}\right)\right] .
$$

Here $\tau_{t: t+H}$ represents the sequence of actions and their outcomes, a trajectory through state-action space $\left[\left(\boldsymbol{x}_{t+1}, \boldsymbol{u}_{t}\right), \ldots,\left(\boldsymbol{x}_{t+H}, \boldsymbol{u}_{t+H-1}\right)\right]$, from the current state $x_{t}$ up to some planning horizon $H$.

The sequential nature of the problem implies that the best decision in the current step depends on the decisions it can make in subsequent states, which now also have to be considered over the multitude of possible state outcomes, and so on. Due to this branching of the event space, optimal decisions under uncertainty usually have to be sought jointly as a policy function $\pi$ mapping a possible state $\boldsymbol{x}_{t}$ to an action $\boldsymbol{u}_{t}$ such that $\boldsymbol{u}_{t}=\pi\left(\boldsymbol{x}_{t}\right)$. Unfortunately, finding an optimal policy $\pi(x)$ for the high-dimensional real-valued state and action spaces of robots is in the general case computationally intractable, both in theory and practice. While the formulation above serves as an introductionary example, we defer further details to Chapter 3.

In addition we often want to solve this problem under constraints on the motion of the robot, which can be written in the form $g\left(\tau_{t: t+H}\right) \geq \mathbf{0}$, where $g($.$) is some arbitrary function of the robot's state and actions. This can in-$ clude requirements on the task the robot has to solve, for example a speed limit. However, due to the risks involved with real autonomous robots, the main focus here is on the safety of the robot and any human bystanders. While one can also include this as a large cost instead of a constraint, it can be difficult to put a number on the cost of a collision that risks human injury. 
Further, due to the uncertainty in our problem we can only satisfy constraints with some high probability $p$. This finally results in the probabilistically constrained, or chance-constrained, sequential decision problem

$$
\begin{array}{ll}
\underset{\pi(\boldsymbol{x})}{\arg \min } & \mathbb{E}_{\boldsymbol{x}_{t: t+H}}\left[c\left(\tau_{t: t+H}\right)\right] \\
\text { subject to } & \\
& \operatorname{Pr}\left(\boldsymbol{g}\left(\tau_{t: t+H}\right) \geq \mathbf{0}\right)>p .
\end{array}
$$

A recurring class of such problems that we study in this thesis is safe navigation for autonomous robots while avoiding collisions with humans working in close proximity, such as in a warehouse, office, home or even outdoor scenarios. A large degree of uncertainty here stems from the difficulty of predicting human behavior in its environment. Regardless of the source of uncertainty, we want to satisfy such safety constraints with high probability.

Since such safe decision-making under uncertainty in 1.3 is computationally infeasible for all but the smallest problems, while robots in the real world have to make decisions in real-time, the decision problem has to be simplified or approximated in practice. In the simplest case, robot behavior may be manually engineered in advance for each situation its designers expect to arise. Unfortunately, this is both difficult and fragile in the openended environments of autonomous robots. More automatic methods from optimization-based planning and control are therefore seeing increased use in robotics. However, these often solve a version of the problem without uncertainty and have to rely on a number of assumptions to guarantee safety.

\section{Research Questions}

In this thesis we examine how recent techniques from machine learning can be used to improve real-time decision-making under uncertainty for autonomous robots. We propose and evaluate a number of techniques to answer what can be broadly classified into two research questions pertaining to their use with real autonomous robots:

RQ1 How can robots learn real-time approximations to policy $\pi(x)$ in 1.3 that are safe under uncertainty.

RQ2 How can robots learn better internal models $p\left(\boldsymbol{x}_{t+1} \mid \boldsymbol{x}_{t}, \boldsymbol{u}_{t}\right)$ for improved real-time decision making in 1.3 .

Machine learning has been a major driving force behind the optimism for AI over the last decade. By enabling machines to learn from data, the task of encoding correct behavior of an AI can be automated or greatly eased. With the aid of big data sets, this has resulted in rapid advances for software agents in a wide variety of domains. The strength of machine learning is its generality, it can learn to approximate nearly any task given sufficient data. 
However, being embodied agents in the real-world, robots also pose a number of difficulties for machine learning. These include real-time requirements with limited computational resources, the cost and effort of operating and collecting data with real robots, as well as safety issues for both the robot and human bystanders.

While machine learning is general by nature, overcoming the difficulties with real-world robots outlined above remains a challenge. In this thesis we therefore look for a middle ground on robot learning, leveraging the strengths of both data-driven machine learning and engineering techniques from robotics and control, to find safe approximations in decision making for real robots. This includes combing data-driven world models with realtime planning techniques, using machine learning to improve risk handling in problems of high uncertainty, as well as using machine learning to find computationally efficient and safe policy approximations for use on small embedded systems.

\section{Delimitations}

As the decision problem in (1.3) is very general and computationally intractable, we make a number of delimitations based on the needs of autonomous robots.

D1 We do not require decisions to be optimal, only effective approximations that satisfy desired safe constraints.

D2 While some of the proposed methods can learn in real-time, we only require that methods converge to safe policies that can execute in realtime.

D3 We only require solutions capable of real-time operation on a real robot, not formal guarantees of hard real-time. Safety constraints are expected to hold regardless.

D4 We use statistical notions of probabilistic safety instead of provable absolute safety. This is in part due to limitations of our numerical methods, but also because proving absolute safety of an autonomous robot may require making strong assumptions on the real world.

Safety, learning and autonomy is a particularly difficult combination that can even be impossible for fragile robots without strong assumptions. We therefore chose to primarily focus on the use of learning techniques as a tool for robot engineers. To enable them to create robots that make safe decisions in real-world uncertain environments, which may include use of simulation for training. 


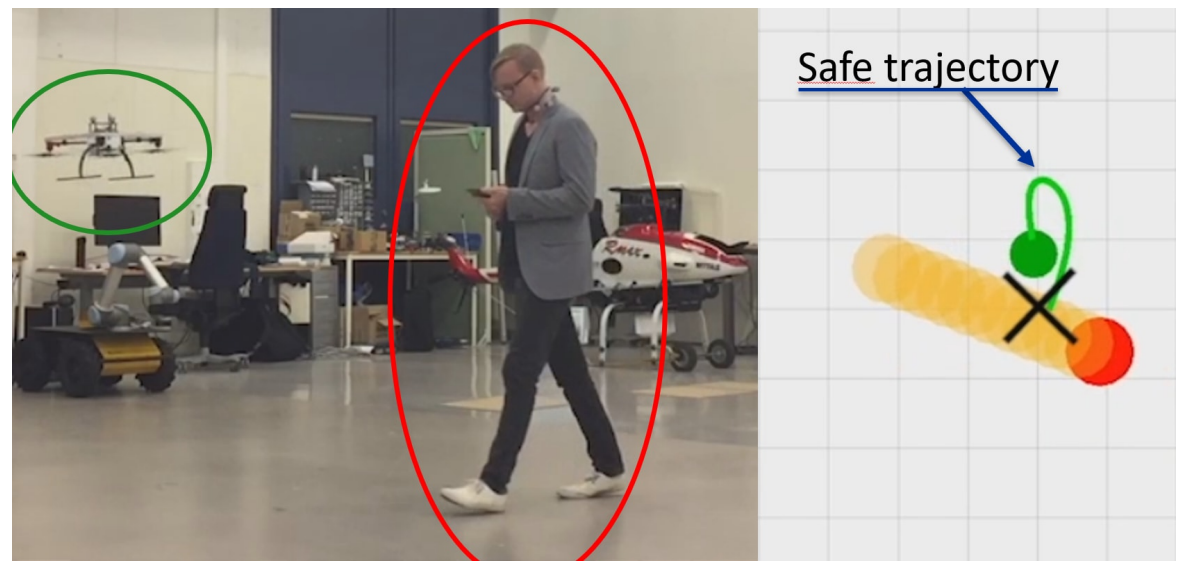

Figure 1.2: An obstacle avoidance experiment with the author playing the role of an inattentive pedestrian heading towards the robot, which has to come up with a safe evasive motion.

\subsection{Methodology}

Several applications in autonomous navigation were used to guide the research in this thesis, with a focus on problems in obstacle avoidance and motion planning under uncertainty. Throughout the course of this thesis, significant effort was also put into evaluating the proposed methods with real robots or reasonable simulations thereof.

The research was conducted in the field robotics group of the Artificial Intelligence and Integrated Computer Systems (AIICS) division at Linköping University, specializing in autonomous unmanned aerial vehicles (UAVs). We primarily worked with ground-level scenarios such as navigating inside buildings, scouting under the canopy of forests, or other ground-level inspection tasks. UAVs in these settings have to face much of the same problems with safety under uncertainty from people as autonomous cars do, exemplified by an obstacle avoidance experiment in Figure 1.2

While the dangers posed to humans by mid-size quadcopters are smaller than for cars, they are still capable of causing injury. They also have stricter limitations on weight and power usage for sensor and computation equipment, which poses additional challenge. Further, such unstructured environments introduces even greater uncertainty than driving situations where traffic rules impose structure on the problem, guiding predictions and behavior. The motion of pedestrians in less structured environments is even more difficult to predict. Additionally, UAVs are constantly at the mercy of wind outdoors. Being both fragile and of limited computational capacity, UAVs can be said to embody the characteristic challenges of autonomous robots, posing a difficult problem especially for learning algorithms. 


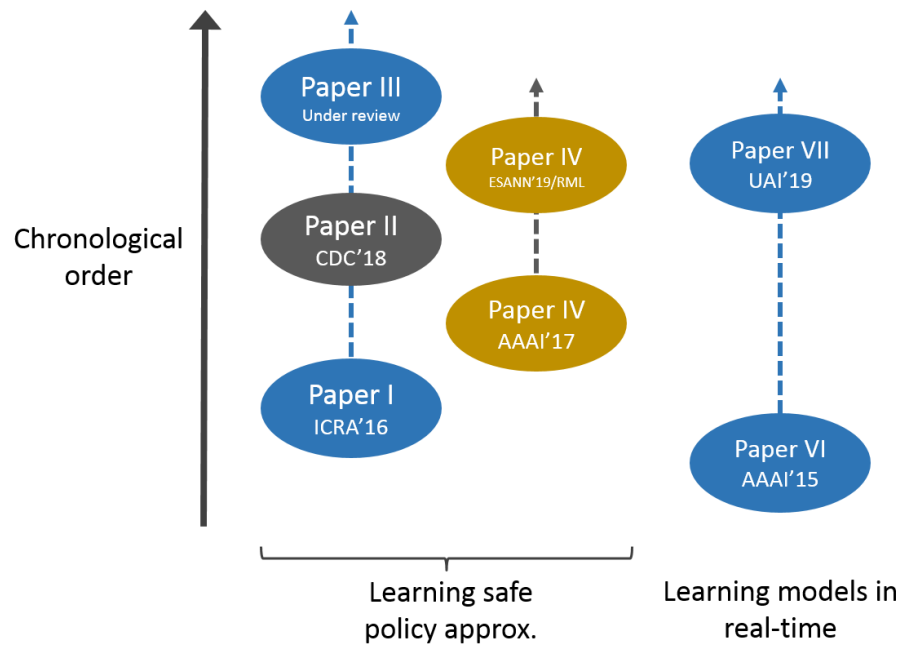

Figure 1.3: The publications in this thesis can be grouped into three research threads, the first two addressing RQ1 and the third addressing RQ2. Blue publications draw on probabilistic machine learning, while gold colored publications draw on deep learning and neural networks. Paper II did not consider learning, but it was combined with learning in Paper III.

To address the research questions, a number of different machine learning techniques were evaluated. Here we focus on two popular areas in machine learning, probabilistic machine learning and deep learning. We use these either on their own, attempting to learn from scratch, or more commonly in various configurations with existing planning and optimization-based methods from robotics and control. Being an inherently cross-disciplinary endeavor, we submitted the research to peer-reviewed top venues across AI, robotics and control.

Several types of quadcopters were used for experiments with these methods. Ranging from the tiny Bitcraz $\bigoplus^{1}$ Crazyflie used to examine computational advantages of neural network approximations, to the internally developed LinkQuad used for obstacle avoidance in Figure 1.2 . and finally a DJ $\mathrm{L}^{2}$ Matrice 100 mounted with lidar and stereo cameras. This final one was also used for the WARA-PS 3 live research demo at Gränsö Slott shown on the cover of this thesis.

\footnotetext{
${ }^{1}$ www.bitcraze.io

2 WwW.dji.com

${ }^{3}$ WASP Research Arena for Public Safety, as part of the Wallenberg AI, Autonomous Systems and Software Program.
} 


\subsection{Brief Outline of Contributions}

All the contributions in this thesis can be seen as finding tractable approximations to different parts of the decision problem under uncertainty in (1.3), attempting to learn approximations that satisfy the computational and safety requirements of autonomous robots in real-world dynamic uncertain environments (DUE). As depicted in Figure 1.3, we can loosely group the research into three threads:

i) Learning risk-adjustments for trajectory planning in DUE

ii) Learning neural-network decision policies for DUE

iii) Learning world models in real-time to reduce uncertainty in DUE

Rather than present three parallel threads chronologically, we have chosen to present each thread sequentially. The contributions are briefly outlined below. Additional detail and context is provided in the following chapters, culminating with the full summary of contributions in Chapter 4 .

Paper I Olov Andersson, Mariusz Wzorek, Piotr Rudol, and Patrick Doherty (2016). "Model-Predictive Control with Stochastic Collision Avoidance Using Bayesian Policy Optimization". In: 2016 IEEE International Conference on Robotics and Automation (ICRA).

Here we consider obstacle avoidance with local trajectory solvers and address how to handle safety constraints for problems with inherent uncertainty, such as the future motion of people. We learn constraints that satisfy a desired level of probabilistic safety via a policy search based on constrained Bayesian optimization. The approach is demonstrated in simulation as well as with a real-quadcopter.

Paper II Olov Andersson*, Oskar Ljungqvist*, Mattias Tiger*, Daniel Axehill, Fredrik Heintz (2018). "Receding-Horizon Lattice-Based Motion Planning with Dynamic Obstacle Avoidance". In: Proceedings of IEEE 57th Annual Conference on Decision and Control (CDC).

In this collaboration we consider complex in-door environments with moving obstacles. By pre-computing dynamically-feasible lattice approximations using optimal control, we leverage graph search for realtime spatio-temporal motion planning in 3D for quadcopters.

Paper III Olov Andersson and Patrick Doherty, "Learning Safe Trajectory Planning in Dynamic Uncertain Environments", Journal article under review. In this journal article we generalize the constraint learning technique in Paper I to learn risk adjustments for the general motion planning problem under uncertainty. We demonstrate this not only on motion 
uncertainty in local obstacle avoidance, but by extending the planner from Paper II to learn risks induced by limited visibility.

Paper IV Olov Andersson, Mariusz Wzorek, and Patrick Doherty (2017). "Deep Learning Quadcopter Control via Risk-Aware Active Learning". In: Proceedings of the Thirty-First AAAI Conference on Artificial Intelligence (AAAI).

We explore computational advantages of deep-neural-network policy approximations, where model-predictive control is used as a teacher, and knowledge of safety constraints is used to improve the safety of the approximation. The approach is demonstrated with a learned obstacle avoidance behavior, in simulations as well as by implementation onboard a real $7 \mathrm{~cm}$ quadcopter.

Paper V Olov Andersson and Patrick Doherty (2019). "Deep RL for Autonomous Robots: Limitations and Safety Challenges". European Symposium on Neural Networks. An earlier version was presented in the ICML'18 Workshop on Reproducibility in ML.

We evaluate a popular deep reinforcement learning approach on an obstacle avoidance domain with dynamic and uncertain obstacle motion. We identify problems with convergence to safe policies on environments with inherent uncertainty and local minima, as well as discuss its implications for use in autonomous robots.

Paper VI Olov Andersson, Fredrik Heintz, and Patrick Doherty (2015). “ModelBased Reinforcement Learning in Continuous Environments Using Real-Time Constrained Optimization". In: Proceedings of the TwentyNinth AAAI Conference on Artificial Intelligence (AAAI).

In our chronologically first paper, we started from the reinforcement learning problem and proposed a real-time approximation by combining sparse Gaussian process models with recent model-predictive control solvers. This attempted to address the case where we want to incorporate safety constraints and the robot dynamics are a priori unknown but deterministic.

Paper VII Olov Andersson*, Per Sidén*, Johan Dahlin, Patrick Doherty, Mattias Villani (2019). "Real-Time Robotic Search using Structural Spatial Point Processes". In:Proceedings of the Thirty-FifthConference on Uncertainty in Artificial Intelligence (UAI).

We learn a structured spatial model for quadcopter victim search after disasters to find search plans that minimize victim harm. To satisfy the real-time requirements of the application, we leverage an Iterative Nested Laplace Approximation (INLA) for Bayesian learning from both GIS priors and real-time data, as well as solve the planning problem via a Monte-Carlo tree search tailored to the problem. 


\subsection{Thesis Outline}

The rest of the thesis is structured as follows. In Chapter 2 we briefly cover statistical inference and the supervised learning techniques used throughout the thesis. This includes learning models of robot dynamics, cost surfaces for Bayesian optimization as well as deep neural network policy approximations. In Chapter 3 we summarize the prerequisite background for the optimization techniques used to solve the decision problem in (1.3) and put them in the context of related work. Finally, Chapter 4 concludes with a summary of the contributions and explores promising avenues for future work. The final part of this thesis includes the relevant publications. 

ChAPter

\section{Learning and Inference}

This chapter will give a brief introduction to statistical learning and inference. As the literature on this subject is vast, this chapter is not intended as an exhaustive overview. The aim is only to provide context for the techniques used in this thesis.

\subsection{Preliminaries}

We take a probabilistic view of machine learning. This has the advantage of giving succinct definitions with the rigor of probability theory. Most other approaches can be seen as different approximations to full probabilistic inference. Here we only present a high level overview, for more details we refer to Bishop (2006) for a machine learning perspective, and Gelman et al. (2003) for a perspective from Bayesian statistics. In the following we assume some basic knowledge of probability theory. For brevity, we sometimes casually refer to probability mass and density functions as probability distributions and assume use of appropriate measure. We also make use of the common shorthand of writing $p(x)$ instead of $\operatorname{Pr}(X=x)$.

In the probabilistic view, we assume there is some probability distribution $p\left(x_{1}, \ldots, x_{n}\right)$ over all variables of interest $X_{1}, \ldots, X_{n}$. This can include both robot and world state based on noisy sensor readings, but also any model parameters that need to be learned. Learning is then reduced to inference in a probabilistic model.

To facilitate inference we primarily rely on two rules derived from probability theory. Assume that we have some partitioning of the set of variables into two partitions $a$ and $b$, each representing one or more variables $X_{i}$.

The marginalization rule takes a joint distribution $p(a, b)$ and marginalizes out nuisance variables $b$ that are not of interest, to produce a marginal distri- 
bution only over $a$. Intuitively, this can be understood as summing or integrating over all outcomes of nuisance variables $b$ for the remaining variables in $a$,

$$
p(a)=\int p(a, b) d b .
$$

The product rule, sometimes called the chain rule of probability, allows you to factorize any joint probability distribution into a product of conditional and marginal distributions,

$$
p(a, b)=p(a \mid b) p(b) .
$$

This is very useful because defining a joint probability distribution directly can be difficult. Particularly for discrete variables, due to the joint outcome space being the Cartesian product of all included variables. Many parts of a model are often more naturally expressed as conditional distributions and simple prior distributions. This also allows use of any known conditional independence, such that $p(a \mid b, c)=p(a \mid b)$, which can greatly simplify the model. For models with complicated structure, these independence assumptions can be made explicit by e.g. defining them via a probabilistic graphical model, such as a Bayesian network (Koller and Friedman, 2009).

In addition, when we want to solve decision problems where the cost of a choice depends on uncertain variables, you generally define it in term of expected values. These represent the average cost over all possible outcomes of the unknown variables, weighted by their respective probability

$$
\mathbb{E}_{p(a)}[g(a)]=\int g(a) p(a) d a,
$$

where $g(a)$ is allowed to be an arbitrary function of the uncertain variables, and subscript $p(a)$, or sometimes just subscript $a$, makes explicit which uncertain variables we are taking the expected value over.

\subsection{Learning and Parameteric Models}

Probabilistic models are typically used to make inferences from data. In robotics the data is usually collected from noisy sensor readings and we are interested in making inferences about the surrounding environment or the robot itself. A model is constructed as a joint probability distribution, suitably factorized using the product rule, where some variables may be observed and others are uncertain. Assuming that variables in partition $b$ are observed we want to compute $p(a \mid b)$, set the observed variables in the joint distribution to their data values and marginalize out any remaining unobserved variables from $a$ that we are not interested in. 
Even when we do not need to marginalize out nuisance variables, we may still need marginalization to normalize the posterior $p(a \mid b)$. To see this, consider a simple supervised learning example where we want to approximate some function $\boldsymbol{y}=f_{\theta}(\boldsymbol{x})$ parameterized by $\theta$, given examples of inputs $\boldsymbol{x}$ and noisy outputs $y$. It is convenient to model the noisy $y$ as a conditional distribution given $\boldsymbol{x}$ and $\theta$, with a prior on $\theta$, resulting in $p(\boldsymbol{y} \mid \boldsymbol{x}, \theta) p(\theta)$. However, what we want is the posterior distribution of $\theta$ conditioned on the data. For this we can use a variant of the eponymous Bayes' rule of Bayesian statistics,

$$
p(\theta \mid \boldsymbol{y}, \boldsymbol{x})=\frac{p(\boldsymbol{y} \mid \boldsymbol{x}, \theta) p(\theta)}{p(\boldsymbol{y} \mid \boldsymbol{x})} .
$$

It is easy to see that Bayes' rule is just an application of the product rule, choosing another way to factorize the distribution $p(\boldsymbol{y}, \theta \mid \boldsymbol{x})$. As noted, the normalizing factor $p(\boldsymbol{y})$ is unknown here and requires marginalization to compute. Here we only considered one example on $x$ and $y$, but in practice we often have multiple examples represented by data matrices $\mathbf{X}$ and $\mathbf{Y}$. In supervised learning it is common to assume that the data likelihood term is independent and identically distributed (i.i.d) for each example, such that $p(\mathbf{Y} \mid \mathbf{X}, \theta)=\prod_{i=1}^{N} p\left(\boldsymbol{y}_{i} \mid \boldsymbol{x}_{i}, \theta\right)$. This assumption may not always hold in robotics but it can still be a useful approximation.

The integrals of marginalization in equation (2.1) or expectations in equation (2.3) often lack a closed-form solution in practice. Approximations generally have to be used when these are non-linear or non-Gaussian. In addition, while the dimensionality of robot state $x$ can be in the range of dozens, to learn advanced models it is not uncommon to require inference over millions of model parameters. High precision approximations such as numerical quadrature are intractable for high-dimensional learning problems, and even sampling can quickly get infeasible under the real-time requirements of robots. Some approximations reduce the inference problem to finding parameters of some simplified family of posterior distributions, but efficiently finding good general-purpose posterior approximations is still very much an active research topic. In paper Paper VII we use an iterative nested Laplace approximation (INLA) for real-time probabilistic inference in a structured spatial model for disaster response. This uses a sequence of Gaussian approximations engineered for that type of latent Gaussian models (Rue, Martino, and Chopin, 2009).

The simple maximum-aposteriori (MAP) appoximation instead optimizes on the posterior distribution and uses a mode as a point estimate $\hat{\theta}$. These are efficient to compute but optimistic due to disregard of parameter uncertainty. By foregoing use of prior, this further reduces to the classical maximum likelihood estimate (MLE). These are ubiquitous in practice, and throughout this thesis we use both of these for parts of models where probabilistic inference lacks closed-form solution or effective approximations. Care must also be taken to avoid poor point estimates. Due to disregard 
of probability mass, in complex models these can be extremely sensitive to small perturbations of the training data, known as overfitting.

Parametric models easily allow you to incorporate prior knowledge. For example in supervised learning of $\boldsymbol{y}=f(\boldsymbol{x})$ we may know the mathematical structure of the function $f$ and only need to estimate a few parameters. However, the structure of some real-world phenomena is difficult or too complicated to derive from prior knowledge. In that case we may want to learn a function with a minimum of assumptions. This is the case in the classical view of reinforcement learning, where the world is treated as entirely unknown. One class of completely data-driven models are Gaussian processes. These are non-parametric in the sense that their complexity can increase with the amount of data. We will later also cover neural network models, which are parametric models that can similarly approximate any function if allowed enough data and parameters. Although it is all just probabilistic inference over parameters in different types of models, one can use parametric and entirely data-driven models to make an informal distinction between uncertain and unknown, reflecting a type of Knightian uncertainty (Knight, 1921). For example, in reinforcement learning the world model $p\left(\boldsymbol{x}_{t+1} \mid \boldsymbol{x}_{t}, \boldsymbol{u}_{t}\right)$ is nearly always assumed entirely unknown.

\subsection{Gaussian Processes}

Gaussian processes are a popular choice of probabilistic non-parametric models where both the predictive mean and variance of $f$ can be analytically inferred from data under some assumptions. Formally, given a training set of $n$ observations on input variables $\boldsymbol{X} \in \mathbb{R}^{d \times n}$ and outputs $\boldsymbol{y} \in \mathbb{R}^{d}$ where y is corrupted by additive noise $y=f(x)+\epsilon$, one can put a Gaussian process prior on the latent function $f(x)$ and attempt to learn $f(x)$ from data.

A Gaussian process (GP) defines function values of $f$ as a set of random variables, any finite number of which have a joint Gaussian distribution (Rasmussen and Williams, 2006). The process is completely specified by a mean function $m(\boldsymbol{x})$ and a covariance function $k\left(\boldsymbol{x}, \boldsymbol{x}^{\prime}\right)$ that are functions of the input variables. For clarity we assume that all data is standardized with mean zero, turning the covariance function into

$$
k\left(x, x^{\prime}\right)=\mathbb{E}\left[f(x) f\left(x^{\prime}\right)\right] .
$$

This defines the covariance of function values at pairs of input points, such that the distribution of any points on $f(\boldsymbol{x})$ is completely specified by the joint multivariate Gaussian.

An example of a common covariance function is the squared-exponential, which decays with the distance between points according to

$$
k_{f}\left(x, x^{\prime}\right)=\sigma_{f}^{2} \exp \left[-\frac{1}{2} \sum_{i=1}^{d}\left(\frac{x_{i}-x_{i}^{\prime}}{\ell_{i}}\right)^{2}\right] \text {. }
$$


The parameters $\sigma_{f}^{2}$ and $\ell_{i}$ represent signal variance and per-dimension length-scales respectively, which together make up the hyperparameters $\boldsymbol{\theta}_{f}$. As the hyperparameter posterior lacks closed-form solution, either sampling or MAP inference is used for that part of the model instead. The length-scales can adapt to attenuate unneeded input dimensions in the problem, known as automatic relevance determination. We also make heavy use of the Matérn family which adds a smoothness parameter $v$ to the covariance function, see Ch.4 of Rasmussen and Williams (2006) for a discussion of why this is desirable.

The covariance function is used to define matrices of covariances between function values for the input points $X$, any set of prediction points $X_{*}$ as well as their cross-covariances. We denote these as $\Sigma_{X, X}, \Sigma_{X_{*}, X_{*}}$ and $\Sigma_{X, X_{*}}$ respectively. As points on $p\left(f(x) \mid \boldsymbol{X}, \boldsymbol{\theta}_{f}\right)$ are multivariate Gaussian, when the observation noise is also Gaussian with standard deviation $\sigma_{n}^{2}$, the data likelihood $p\left(\boldsymbol{y} \mid \boldsymbol{X}, \boldsymbol{\theta}_{f}, \sigma_{n}^{2}\right)$ is jointly Gaussian with covariance $\boldsymbol{\Sigma}_{\boldsymbol{X}, \boldsymbol{X}}+\sigma_{n}^{2} I$, and the predictive distribution at any new points $\boldsymbol{x}_{*}$ admit closed form solution. Although a Gaussian observation model is suited to the real-valued regression tasks often encountered in robotics, other observation models do not necessarily admit a simple closed-form solution. For example, the number of people observed in the disaster response model from Paper VII are assumed Poisson distributed, where we rely on a numerical INLA approximation to allow real-time Bayesian inference.

The non-parametric nature of Gaussian processes, coupled with closedform of approximate probabilistic inference on $f(x)$, makes them very flexible models while remaining resistant to overfitting even for small data sets. Data efficiency is highly desirable for robotics due to the cost of operating and maintaining real hardware. Unfortunately, the non-parametric nature of GPs means that computational complexity of training and prediction also increases with the number of data points, being $O\left(n^{3}\right)$ and $O(n)$ respectively. This quickly gets infeasible even on a desktop computer, and real robots tend to have more limited resources. Much effort has been invested in finding cheaper sparse approximations, and this is still an active research area. Such sparse processes, visualized in Figure 2.1. use a small number of inducing inputs as anchor points for an approximation while still aiming to retain reasonable accuracy.

Most of our contributions related to GPs are in the context of approximations to the decision problem in (1.3), which will be further described in Chapter 3 In Paper I and Paper III we rely on a Gaussian-process based Bayesian optimization to learn safe policies under probabilistic safety constraints. Here the objective and constraint surfaces are modelled by GPs to intelligently sample points during global optimization. In two papers we additionally focused on learning models of the environment using variants of Gaussian processes. In Paper VI, we suggest a novel constrained reinforce- 


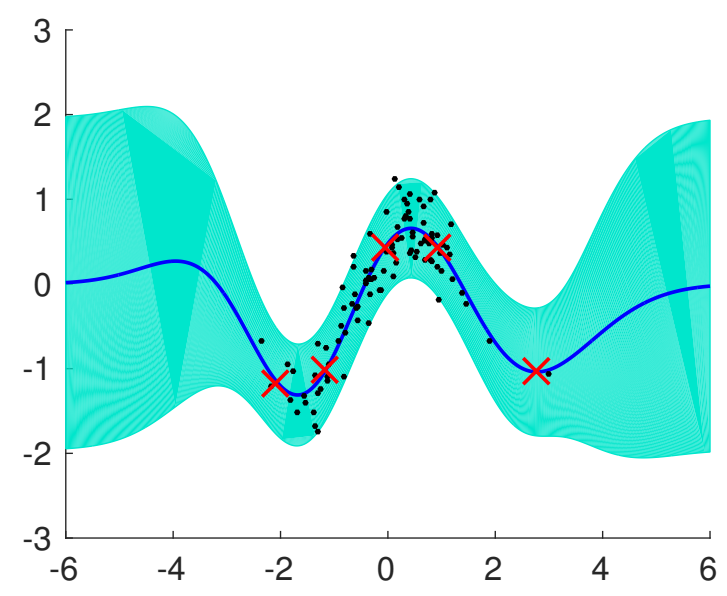

Figure 2.1: Sparse approximation to a $f: \mathbb{R}^{1} \rightarrow \mathbb{R}^{1}$ Gaussian process inferred from data points in black. Predicted mean and $95 \%$ probability intervals in blue and cyan respectively. The red crosses represent the inducing points of the sparse approximation.

ment learning method using sparse GPs to efficiently learn world models. We use the FITC approximation (Snelson and Ghahramani, 2006) which has been shown to work well with the square-exponential kernel in applications, and has a computational complexity of $O\left(\mathrm{~nm}^{2}\right)$ and $O(\mathrm{~m})$ for training and prediction respectively. In Paper VII we learn structured spatial models related to Gaussian processes in a disaster response application, relying on the aforementioned INLA approximation to handle the non-Gaussian parts.

\subsection{Deep Learning}

While Gaussian processes are highly accurate for small data sets, and sparse approximations can handle larger ones, training and prediction with very large data sets can still be a problem. Even more importantly, the generalization ability of "black-box" covariance functions like the popular squareexponential scale poorly with input dimension. Unless automatic relevance determination can reduce the problem to only a moderate number of relevant input variables, learning a Gaussian process may simply not be practical. Deep learning attempts to address this problem by learning hierarchical, so called deep, representations. The, by far, most popular models are multilayered neural networks (Goodfellow, Bengio, and Courville, 2016). These are composed of layers of parameterized artificial neurons, each a linear combination of its inputs passed through a non-linear activation function. Network structure can range from generic fully-connected feed-forward networks exemplified by Figure 2.2. to variants imposing additional structural 


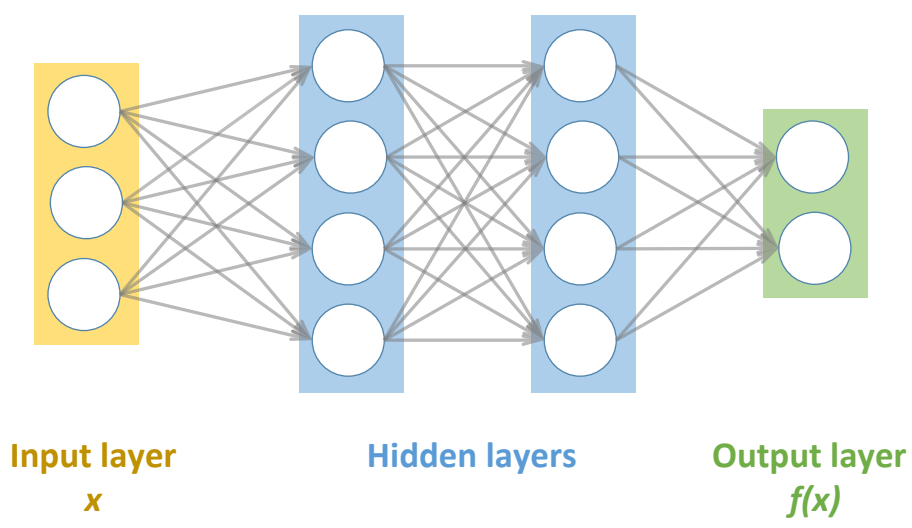

Figure 2.2: Architecture of a fully-connected neural network with two hidden layers for learning some function $f: \mathbb{R}^{3} \rightarrow \mathbb{R}^{2}$. Artificial neurons are shown in white and their inputs are represented by arrows.

assumptions tailored to a particular domain, such as images or text. The idea is that a hierarchical representation should be able to naturally learn abstractions to better exploit existing structure in the task, and therefore decompose the problem into a composition of smaller ones. Mathematically, this can be seen as learning a composition of functions $f(g(h(\boldsymbol{x})))$, where hopefully no individual function in the composition has to be as complicated as a "flat" representation $f(\boldsymbol{x})$ would be.

While technically parametric, if given a sufficient number of parameters, they are arbitrarily flexible black-box models similar to Gaussian processes. However, parameter inference for such deep architectures usually lack a closed-form solution, and point estimates typically have to be found via optimization. A great advantage of deep learning is the existence of efficient software for offloading training to a GPU, allowing for larger data sets and models than may have previously been practical. We use Tensorflow (Abadi et al., 2016), a popular graph-based language for numerical computation from Google.

In Paper IV we use a variant of deep supervised learning to learn fast policy approximations $\hat{\pi}_{\theta}(x)$ for the agent decision problem in 1.3), which can be helpful to satisfy real-time requirements on computationally limited robotic systems. Our approach relies on a trajectory solver such as in Paper I to generate large quantities of training data, with a resampling technique to focus on safety constraints. In Paper V we instead examine directly learning neural network policies via deep reinforcement learning, which we will cover in Chapter 3 .

In both cases we use fully-connected, sometimes called dense, feedforward deep neural networks $(\mathrm{DNN})$, with the parameter vector $\theta$ representing the weights $\boldsymbol{W}_{i}$ and biases $\boldsymbol{b}_{i}$ of neurons for each layer $i$. 
Each DNN layer $i$ was defined by

$$
\boldsymbol{y}_{i+1}=\boldsymbol{h}_{i}\left(\boldsymbol{W}_{i} \boldsymbol{y}_{i}+\boldsymbol{b}_{i}\right)
$$

with network input layer $\boldsymbol{y}_{1}=\boldsymbol{x}$, output layer $\boldsymbol{y}_{N}=\hat{\pi}(\boldsymbol{x})$ and $\boldsymbol{h}_{i}(x)$ is the (vector) activation function for layer $i$. We use the popular ReLU activation function, which for each neuron $j$ is the scalar function

$$
h_{i, j}(x)= \begin{cases}x & \text { if } x>0 \\ 0 & \text { otherwise }\end{cases}
$$

for all hidden layers $i$, except the output layer $\boldsymbol{y}_{N}$, which is linear.

Deep neural network training is currently an area under intense research. Most rely on a number of approximations and tricks, such as stochastic gradient approximations from mini-batches to better scale with large data sets. For supervised learning we found adaptive momentum (Kingma and Ba, 2015) useful, and used dropout (Srivastava et al., 2014) to mitigate overfitting. While the training was done offline on a GPU, we managed to implement the resulting network from Paper IV for real-time use on a nanoquadcopter microcontroller. Due to the rising trend of GPU miniaturization for embedded systems, we expect such methods to be of increasing viability for robotics. 
ChAPter

\section{3 \\ Decision, Planning and Control under Uncertainty}

Here we provide a brief overview of different approaches to sequential decision making under uncertainty and their application to planning and control for autonomous robots. As noted in Chapter 1, there is a large body of work on solving sequential decision problems, crossing over into several different fields. We do not aim to give a complete account of this body of work here, only to provide context for the publications in this thesis. We refer the interested reader to Thrun, Burgard, and Fox (2005a) and Deisenroth, Neumann, Peters, et al. (2013) for a robotics perspective. A thorough treatment from the control perspective can be found in Bertsekas (2005) and Bertsekas (2012), while Powell (2007) consider operations research. Finally, a classical introduction to reinforcement learning in discrete domains can be found in Sutton and Barto (2018).

The rest of this chapter is structured as follows. First we provide a recapitulation of decision-making under uncertainty from the introduction. Then we provide a taxonomy of different types of decision problems, introduce relevant solution methods, and then connect them to related techniques in robotics and control, leading up to a summary our contributions in Chapter 4 .

\subsection{Making Safe Decisions Under Uncertainty}

We want autonomous robots to navigate the dynamic uncertain environments of the real world, which can be formalized as solving sequential decision problems under uncertainty,

$$
\underset{\pi(x)}{\arg \min } \mathbb{E}_{x_{t: t+H}}\left[c\left(\tau_{t: t+H}\right)\right] .
$$


Here $\tau_{t: t+H}$ represents a trajectory through state-action space $\left[\left(\boldsymbol{x}_{t+1}, \boldsymbol{u}_{t}\right), \ldots,\left(\boldsymbol{x}_{t+H}, \boldsymbol{u}_{t+H-1}\right)\right]$, from the current joint robot and world state $x_{t} \in \mathcal{X}$, via chosen robot actions $\boldsymbol{u}_{t} \in \mathcal{U}$, over some planning horizon $H$. As continuous-time problems can be transcribed into discrete time via numerical integration, we here only consider discrete-time problems. The world state therefore evolves in discrete time steps $x_{t}$ according to some probability distribution $p\left(\boldsymbol{x}_{t+1} \mid \boldsymbol{x}_{t}, \boldsymbol{u}_{t}\right)$. The uncertain nature of this world transition model can result from imperfect models of robot actuation or other parts of the environment, including human behavior. In practice, all current and future state also carry uncertainty from noisy sensor observations $\boldsymbol{o}_{t}$ used to infer the state via some observation model $p\left(\boldsymbol{o}_{t} \mid \boldsymbol{x}_{t}\right)$, which for ease of exposition we defer to discuss until later. Taken together, this results in a probability distribution over future state based on action choices. The sequential nature of the problem implies that the best decision in the current step depends on the decisions it can make in subsequent states, which now also have to be considered over the multitude of possible state outcomes, and so on. Due to this branching of the event space, optimal decisions usually have to be sought jointly as a function of the state, such that $\boldsymbol{u}_{t}=\pi\left(\boldsymbol{x}_{t}\right)$. For brevity, we here make the assumption that such a decision policy $\pi(\boldsymbol{x})$ is invariant of time. Finding an optimal policy for non-trivial state and action spaces is computationally very challenging.

In addition we want to solve this problem under constraints. While this may include arbitrary task requirements such as a speed limit, due to the fragility and dangers involved with real robots, the main focus here is on safety of the robot and human bystanders. Due to state uncertainty, we can only satisfy such safety constraints with high probability. This finally results in the probabilistically constrained, or chance-constrained, sequential decision problem from (1.3) revisited,

$$
\underset{\pi(x)}{\arg \min } \quad \mathbb{E}_{x_{t: t+H}}\left[c\left(\tau_{t: t+H}\right)\right]
$$

subject to

$$
\operatorname{Pr}\left(g\left(\tau_{t: t+H}\right) \geq 0\right)>p .
$$

A particular class of such problems that we study in this thesis is avoiding collisions with humans working in close proximity, such as in a warehouse, office, home or even autonomous outdoor scenarios. The uncertainty here largely stems from the difficulty of predicting human behavior, and we want to satisfy safety constraints with high probability. Since we exclusively consider applications of such safe decision making for autonomous robots, we further seek approximations $t \&(1 / 3)$ that are feasible to run in real-time on real robots while still satisfying the desired safety constraints. 


\section{A Note on Optimality}

We here make a distinction between optimal behavior, and the optimizationbased methods we use in this thesis. Our aim is to learn computationally feasible approximations to planning and control under uncertainty for autonomous robots in the real world. We approach this from an AI and machine learning perspective. By contrast, classical optimal control techniques put more focus on optimality and hard constraints, which may not be feasible to achieve or prove in uncertain environments. We further argue that optimality may not be as desirable because the objective function is itself also an approximation in most cases. For example, exactly by which criteria should a future household robot tasked with doing dishes and cleaning be judged? Further, subtle issues can arise even in basic navigation tasks of getting from place A to B. A time-optimal humanoid robot navigating an office may go running through corridors. Even when it is risk-free, humans rarely do this even when in a hurry, as even simple motion is governed by complex factors such as social norms. One solution may be to learn the social norms through the cost or reward function, presumably like humans do. Many classical reinforcement learning techniques also assume unknown reward function (Sutton and Barto, 1998), but this is rarely used with real robots due to the increased data requirements this implies. We leave such human-robot considerations for future work and just assume we have some reasonable but approximate objective towards which we want to optimize. The focus here is instead on finding real-time best-effort solutions that satisfy desired safety constraints.

While this may seem like a strong limitation, agent behavior only needs to be adequate for its real world task. Consider that humans sometimes make demonstrably poor decisions, and therefore are not optimal either. While humans are often modeled as rational agents in the social sciences, it has long been known that human rationality is bounded (Simon, 1955), although the exact mechanics appear difficult to pin down. While biological plausibility is not an objective of this thesis, this is a strong indication that optimality can be seen as more of an ambition rather than a strict requirement.

\subsection{Taxonomy of Sequential Decision Making}

Sequential decision problems come in many variants, where some differ only in notation. For example, in classical reinforcement learning it is customary to define the objective function of the agent in terms of maximizing rewards or utility instead of minimizing costs, which is mathematically equivalent. Likewise, classical reinforcement learning originated with discrete problems, where states $s \in \mathcal{S}$ and actions $a \in \mathcal{A}$ are discrete sets. Robot sensors and actuators are better represented by metric spaces, and therefore it is common to define these as real vectors for state $x$, and actions as real-valued con- 
trol inputs $u$ instead. Throughout the publications in this thesis we sometimes use these definitions interchangeably. A common formalism is that of Markov Decision Processes (MDP). Such decision processes are assumed to satisfy the Markov property, that state transitions are conditionally independent of all earlier state $p\left(\boldsymbol{x}_{t+1} \mid \boldsymbol{u}_{t}, \boldsymbol{x}_{t}, \ldots, \boldsymbol{u}_{1}, \boldsymbol{x}_{0}\right)=p\left(\boldsymbol{x}_{t+1} \mid \boldsymbol{x}_{t}, \boldsymbol{u}_{t}\right)$. We do not always require this, but it is generally a safe assumption to make since a non-Markovian process can be converted to a Markovian process on an augmented state space.

For autonomous robots, the representation of the state-space itself has to be constructed and estimated from imperfect sensors. A common simplification is to simply assume state is perfectly observable, which neglects uncertainty. On the other end of the spectrum, the optimal solution is to model how the robot's belief over the state $x$ changes based on its actions, and plan with regard to this. In some problems an agent has to weigh the value of pure information gathering actions to better solve its task later, against trying to solve it without this information. This is a famously difficult exploration versus exploitation problem. Standard MDP solution techniques can be used to find a solution to such a partially observable MDP (POMDP) via a belief-augmented state space (Thrun, Burgard, and Fox, 2005a). Unfortunately, since the belief is now a probability distribution over the state, this can make the problem dimension exponentially larger. In this thesis we avoid full belief-space planning to retain real-time capability. In Paper I and Paper III we take observation uncertainty into account for safety constraints without doing belief space planning. This works well in domains where state uncertainty is nearly invariant to future action choices. In Paper VII we also solve an information gathering problem in real-time via a determinized approximation to the belief-space.

If the world model $p\left(\boldsymbol{x}_{t+1} \mid \boldsymbol{x}_{t}, \boldsymbol{u}_{t}\right)$ is simultaneously learned as the agent has to make decisions, it technically does introduce an exploration problem. However, instead of seeking an optimal belief-space solution, it is common in applications to learn passively, via randomization or heuristic exploration strategies. In the randomized case, the policy can also be seen as a probability distribution conditioned on the state $\pi(\boldsymbol{u} \mid \boldsymbol{x})$, rather than a deterministic function of the state. In robotics, some parts of the model can likely be derived from physics, although identifying such an uncertain parametric model is still a difficult problem. In reinforcement learning, the model is usually considered entirely unknown and always has to be learned. In this thesis we attempt both approaches, but find that simpler parametric models can be sufficient for our purposes if their uncertainty is also included for decision making. The reinforcement learning problem can be further be decomposed into separate model learning and planning problems, alternatively model-free methods circumvent having an explicit representation of the model to directly compute the policy. An advantage of the former model-based methods is that they tend to be more data efficient, a key desiderata for use on real 
robots. However, learned or derived models can still be used as simulators with model-free methods, which is not uncommon in practice.

The most important delineation is in how we compute the optimal policy - the choice of policy optimization approach. Do we directly search for a policy $\pi(x)$, or do we use dynamic programming to compute a value function $V(x)$ as an intermediate step in finding the policy? These both require finding the optimal action over all relevant states $x$ and are thus universal solutions to the decision problem. If the model is assumed deterministic, one can instead compute a local solution as a trajectory or plan from the current state $\boldsymbol{x}_{t}$, which is simply recomputed online in real-time at each time $t$. Trajectory, and planning methods generally, typically neglect uncertainty, which can be advantageous from a computational perspective. Although this is sub-optimal and potentially unsafe, most popular motion planning and control solutions in robotics rely on these for real-time decision making. Several of our contributions also use these as a basis for learning safe real-time approximations to the decision problem under uncertainty.

Another delineation can be made based on planning horizon. An underlying problem can be episodic with a finite duration or terminal state, or it can be an infinite non-episodic task. Non-episodic tasks are of more interest for behavior in autonomous robots. Yet, even if the task is non-episodic, the planning horizon of the agent, as defined in equation (1.3), only needs to be sufficiently long to solve the underlying tasks. The obstacle avoidance and motion planning applications we consider here typically need 10 to 100 decision points over a planning horizon of seconds to minutes. This is either explicit via a finite horizon $H$ or implicit via an infinite horizon $H \rightarrow \infty$, where the latter requires geometric discounting for the objective to remain finite.

A final dimension is inclusion of constraints such as our safety constraints. These can alternatively be represented by penalties in the objective function, either carefully engineered to ensure they will be satisfied, or automatically adjusted via e.g. Lagrangian methods (Nocedal and Wright, 2006).

As an exhaustive overview of these topics is beyond the scope of this thesis, we will focus on giving sufficient context for the papers in this thesis.

\subsection{Policy Optimization}

The most straightforward approach to solve equation (1.3) is via direct policy search, optimizing directly on the policy $\pi(x)$. If the states and actions are discrete, the policy can be enumerated in a table. If they are non-discrete, the optimal policy can be approximated by a parameteric function $\pi_{\theta}(\boldsymbol{x})$ with parameter vector $\theta$. Non-trivial instances of the fully continuous problems we focus on in this thesis lack closed-form solution. We here instead draw on techniques from machine learning to find approximations to this problem. Unfortunately, common approximations lack global optima guarantees. For 
the policy to be powerful enough to represent a good solution, one may have to use a very complex approximation such as a large neural network. Even optimizing for local minima of $\pi_{\theta}(\boldsymbol{x})$ can be challenging as the expected cost over uncertainty results in difficult high-dimensional integrals. Below we attempt to give a brief overview of policy learning techniques currently popular in machine learning.

With the the rise of deep learning, the currently most active research on learning approximate policies is deep reinforcement learning. However, since the focus of this thesis is decision-making for real autonomous robots, their safety requirements impose additional restrictions that have also led us to develop other representations for the policy than neural networks.

Much classical work on policy search in robotics (Kober and Peters, 2012: Deisenroth, Neumann, Peters, et al., 2013) actually relies on domain knowledge to construct parameterized policies with a smaller number of parameters. Much of these were also typically model-free reinforcement learning methods. Model-free methods can technically sidestep the issue of estimating a world model $p\left(\boldsymbol{x}_{t+1} \mid \boldsymbol{x}_{t}, \boldsymbol{u}\right)$ by numerically evaluating policy performance on sampled state transitions and rewards from the robot in the world. These can also be used with simulations if a world model is available. Due to the large number of world interactions needed for policies with a large number of parameters, this may be advisable since real robot hardware is fragile and has safety concerns. From this perspective, model-free reinforcement learning just becomes a means to compute approximate policies.

\section{Policy Gradient Approaches}

Perhaps the simplest approach is to use finite-difference numerical differentiation or derivative-free optimization techniques on the policy parameters $\theta$. Over the last two decades a large number of more advanced approaches have been proposed, many trying to exploit some structure in the problem.

The classical REINFORCE algorithm (Williams, 1992) exploits that under a stochastic policy, the gradient of policy parameters can be rewritten as an expectation that can be estimated via model-free Monte Carlo simulations. This results in an optimization problem over (parameterized) probability distributions $\pi_{\theta}(\boldsymbol{u} \mid \boldsymbol{x})$,

$$
\underset{\pi_{\theta}(\boldsymbol{u} \mid x)}{\arg \min } \mathbb{E}_{\tau_{t: t+H} \mid \pi_{\theta}}\left[c\left(\tau_{t: t+H}\right)\right]
$$

Such stochastic policies are often seen as an integration of randomized exploration strategies into the policy search problem, usually realized by adding local Gaussian noise on a deterministic policy function like a neural network. Much work builds on this, for example to better leverage the sequential structure (Deisenroth, Neumann, Peters, et al., 2013). Modern extensions often better exploit the geometry of the problem and attempts to 
better regulate the step size (Kakade, 2002: Schulman, Levine, et al., 2015. Schulman, Wolski, et al., 2017).

As previously mentioned, since model-free policy search requires a large number of interactions with the system, most early successes using these online with real robots, instead of in simulation, relied on domain knowledge to craft suitable policies with a moderate number of parameters (Kober and Peters, 2012) and reasonable initial values.

In comparison, model-based approaches do not need to interact with the system to update its policy, and are therefore well-known to be much more data efficient (c.f. Sec. 6, Kober and Peters (2012)). However, they can be difficult to compute in the general case since the unknown model has to be marginalized out. A special case is exploited by PILCO (Deisenroth and Rasmussen, 2011), which under some moderately restrictive assumptions on state, reward function and Gaussian process model, actually can compute policy gradients analytically. Recently, there has also been a surge of interest in using approximate inference techniques for Bayesian neural network models and combining these with analytic policy gradient approaches similar to PILCO (Gal, McAllister, and Rasmussen, 2016). If online learning with completely unknown world model is required, this seems like an interesting direction, but the robustness of these approaches appear unclear at this point (Parmas et al., 2019).

\section{Value Iteration Approaches}

All policy gradient methods above can also get stuck in local minima. Another way to solve the decision problem is via dynamic programming, which under some conditions can find a global optima for policy parameters. While this approach has been dominant in classical reinforcement learning, it has seen less use in robotics due to the difficulties of continuous state and actions. Consider the infinite-horizon case of $H \rightarrow \infty$ from $t=0$ in an MDP with $\lambda$-discounted sum cost,

$$
\underset{\pi(\boldsymbol{x})}{\arg \min } \mathbb{E}_{\boldsymbol{x}_{0: \infty}}\left[\sum_{t=0}^{\infty} \lambda^{t} c\left(\boldsymbol{x}_{t}, \pi\left(\boldsymbol{x}_{t}\right)\right)\right] .
$$

The cost sequence under a fixed policy from any state $x$ converges to a value as a geometric sum for $\lambda<1$. Under MDP assumptions, the value of each state can be represented by a time-invariant global value function $V_{\theta}(\boldsymbol{x})$. Further, the value of the current state can be decomposed into an immediate cost plus the expected value of the next state, $V_{\theta}\left(\boldsymbol{x}_{t}\right)=$ $\mathbb{E}_{x_{t+1} \mid x_{t}}\left[c\left(\boldsymbol{x}_{t}, \pi\left(\boldsymbol{x}_{t}\right)\right)+\lambda V_{\theta}\left(\boldsymbol{x}_{t+1}\right)\right]$. Under a known world transition model $p\left(\boldsymbol{x}_{t+1} \mid \boldsymbol{x}_{t}, \boldsymbol{u}_{t}\right)$, this can be used to compute the value function for a particular policy, policy evaluation, via a fixed-point iteration. Value iteration extends this to directly compute the value function $V_{\theta}^{*}(x)$ of the, similarly time- 
invariant, optimal policy $\pi^{*}(\boldsymbol{x})$ via the fixed-point iterations,

$$
V_{\theta}^{i+1}\left(\boldsymbol{x}_{t}\right)=\min _{\boldsymbol{u}_{t}} \mathbb{E}_{\boldsymbol{x}_{t+1} \mid \boldsymbol{x}_{t}, \boldsymbol{u}_{t}}\left[c\left(\boldsymbol{x}_{t}, \boldsymbol{u}_{t}\right)+\lambda V_{\theta}^{i}\left(\boldsymbol{x}_{t+1}\right)\right] .
$$

The optimal policy itself can then be recovered by local one-step choices on the optimal value function. Solving for the value function can also be viewed as solving a dual to the original policy optimization problem (Kober and Peters, 2012).

Most value function approaches in reinforcement learning are modelfree. Instead of computing the expected value of the next state under an uncertain world model, they sample the world transition by interacting with the world, either in simulation or with a real system. A famous example is Q-learning (Watkins and Dayan, 1992), where the eponymous Q-function is a value function augmented with action, $Q_{\theta}(x, u)$, to allow solving for the minimum action $u$ without using a model. This results in the fixed-point iteration

$$
Q_{\theta}^{i+1}\left(x_{t-1}, u_{t-1}\right)=\min _{\boldsymbol{u}_{t}} c\left(\boldsymbol{x}_{t-1}, \boldsymbol{u}_{t-1}\right)+\lambda Q_{\theta}^{i}\left(\boldsymbol{x}_{t}, \boldsymbol{u}_{t}\right) .
$$

The Q-function is incrementally updated after each sampled transition $\left(\boldsymbol{x}_{t-1}, \boldsymbol{u}_{t-1}, \boldsymbol{x}_{t}\right)$, as if the best action will be chosen in the current step. Qlearning is therefore an off-policy algorithm. It is not required to actually follow the optimal policy and can easily incorporate arbitrary exploratory moves. Efficient exploration is generally of great importance for valuefunction approaches to converge in practice.

A fundamental bottleneck with value-function approaches is in the representation of the value function itself. An exhaustive enumeration of all state is often infeasible, as a naive discretization of continuous state spaces suffer from the curse of dimensionality. A major focus of classical reinforcement learning has been to find approximations to such value functions while maintaining favorable convergence properties of the fixed-point iterations. This has shown itself to be difficult for the general case, and more powerful approximations such as neural networks are known to exhibit convergence issues, see Kober and Peters (2012) for a discussion. While dynamic programming is globally optimal, this is not guaranteed to hold for such approximations.

\section{Deep Reinforcement Learning for Continuous Domains}

With the rise of deep learning, there has been a resurgence of interest in value function approaches with deep neural networks, starting with using deep neural networks for Q-functions (Mnih et al., 2015) to learn video game behavior directly from image inputs. However, robotics typically requires continuous action spaces, which introduces additional difficulties, such as having to numerically solve an optimization problem just to minimize $\boldsymbol{u}$ in (3.4). 
In Lillicrap et al. (2015) deep neural-network value-functions were therefore combined with policy gradient updates to better handle continuous problems, known as deterministic deep policy gradients (DDPG).

While such deep neural-network approaches to value-function approximation is a promising direction with considerable research activity, they have so far not had as much success on continuous domains such as robotics applications. A well-known benchmarking study (Duan et al., 2016) found that while the DDPG algorithm of (Lillicrap et al.,2015) initially improved faster than direct policy gradient approaches, they suffered from erratic convergence and sometimes fell behind more traditional counterparts. Instead policy gradient approaches paired with deep neural networks, such as proximal policy optimization (PPO) by Schulman, Wolski, et al. (2017), have so far seen more success on continuous control domains.

However, there has also been some recent concern over how generalizable deep RL results on the standard control benchmarks really are. Henderson et al. (2018) showed that performance is very fragile to hyperparameter tuning. It has also been shown that recent deep RL algorithms can be outperformed on these domains by a carefully tuned random search (Mania, Guy, and Recht, 2018), and Rajeswaran et al. (2017) argued for more domain randomization in them. This highlights an underappreciated problem with comparing simulation results of policy learning techniques to the tasks faced by real-world autonomous robots. As the difficulty of learning a task depends on the intrinsic dimensionality of the data distribution $p(\tau)$, the problem gets easier if the data is confined to a small sub-space. Under fixed policy $\pi(x)$, simulating a deterministic robot and environment from a fixed starting position will always result in the same trajectory, a one-dimensional data distribution. In the simplest case, it may just have learned a single open-loop trajectory for the simulated environment. However, a real robot cannot predict the world entirely in advance. Autonomous robots usually have to be able to deal with a wider range of possible situations than simulation benchmarks often assume.

In Paper V we therefore evaluate trying to learn neural network policies with the popular PPO algorithm on a simplified variant of an obstacle avoidance problem with randomly moving obstacles. This is interesting because standard RL control benchmarks lack inherent uncertainty. The little randomization that exists is generally in the start state, which is perfectly observable. We discovered some issues in getting PPO to converge to a safe policy on this problem. Most notably, the inherent uncertainty in such environments, as well as local minima in policy space, were identified as problems. These convergence issues are in line with, and may also help explain, similarly poor results for older deep reinforcement learning algorithms on a static navigation problem in (Duan et al., 2016). Unfortunately, asymptotic convergence and safety is rarely tested. Real autonomous robots unfortunately have to act in dynamic and uncertain environments, where factors such as people 
or wind are inherently uncertain, and still satisfy safety requirements. To satisfy safety constraints we have therefore mainly focused on other learning techniques, and leave directly learning neural network policies via deep reinforcement learning to future work.

\subsection{Safe Real-Time Planning Under Uncertainty}

Since automatically learning a good global policy or value function approximation is difficult, it seems reasonable to consider local approximations that plan iteratively in real-time.

It is well-known that the unconstrained decision problem in equation 1.2 can be solved analytically for the special case of sum-quadratic costs and the world transition model $p\left(\boldsymbol{x}_{t+1} \mid \boldsymbol{x}_{t}, \boldsymbol{u}\right)$ being linear with Gaussian noise. This is known as the linear-quadratic-Gaussian regulator (LQG), which due to the separation principle (Åstrom, 1970: Georgiou and Lindquist, 2013) is also optimal under Gaussian observation noise when combined with a Kalman filter. This results in the optimization problem

$$
\underset{\pi_{t}(\boldsymbol{x}), \ldots, \pi_{t+H}(\boldsymbol{x})}{\arg \min } \mathbb{E}_{\boldsymbol{x}_{t: t+H}}\left[\sum_{i=t}^{t+H} c\left(\boldsymbol{x}_{i}, \pi_{i}\left(\boldsymbol{x}_{i}\right)\right)\right] .
$$

The resulting time-variant value functions $V_{t}(\boldsymbol{x})$ and policies $\pi_{t}(\boldsymbol{x})$ are quadratic and linear respectively (Bertsekas, 2005), and dynamic programming can be used to compute each $\pi_{t}(\boldsymbol{x})$ via a backwards recursion from $V_{t+T}(x)$. For ease of exposition we have here, and in the following, assumed that the world transition model is forward-integrated from time $t$ in fixed unit time steps via numerical integration. Differential Dynamic Programming (DDP) is a classical trajectory optimization approach for non-linear sumquadratic systems by means of iterative local quadratic model approximations (Mayne, 1966). The popular iterative LQR (iLQR) instead makes local linear model approximations and is computationally cheaper at $O\left(d^{3} H\right)$ per iteration (Todorov and $\mathrm{Li}, 2004$ ), where $d$ is the dimension of the state space. These have been popular for robot learning and control (Abbeel, Coates, and $\mathrm{Ng}$, 2010; Tassa, Erez, and Todorov, 2012).

However, they do have three downsides. The most obvious is that their trajectories and local-linear control policies will need to be recomputed in real-time unless you only need to track canned trajectories in static environments. Sometimes relying on such local-linear feedback laws around canned trajectories is sufficient, which has been used to great effect for mimicking aerobatics maneuvers (Abbeel, Coates, and $\mathrm{Ng}$, 2010). However, contrary to most policy approaches they are also computationally tractable to compute, or at least to find a local minima. 
A second problem is that these are not actually correct under uncertainty once we leave the LQG assumptions of linear model, quadratic objective and Gaussian noise models.

The final problem is that when computing behavior for real robots we want to incorporate safety constraints, for example to avoid falling over, or colliding with human bystanders. Usually there is some state we want the robot to reach, and regions of the state space we want it to avoid. Unfortunately, such constraints generally ruin the simple quadratic value function above.

In numerical optimal control, it is common to instead optimize trajectories for assumed deterministic problems with constraints. There is a host of mature solvers for these, ranging from general-purpose non-linear programming (NLP) solvers from the optimization community (Nocedal and Wright, 2006), to highly specialized model-predictive control (MPC) solvers from the control community. MPC solvers are highly-optimized for real-time use, typically operating in a receding-horizon fashion with a fixed planning horizon $H$ and warm-starts from the previously optimal trajectory. This turns the policy optimization problem on an uncertain trajectory distribution in equation (1.3) into a deterministic trajectory optimization problem,

$$
\begin{array}{cl}
\underset{\tau_{t: t+H}}{\arg \min } & c\left(\tau_{t: t+H}\right) \\
\text { subject to } & \\
& \boldsymbol{x}_{i+1}=\boldsymbol{f}\left(\boldsymbol{x}_{i}, \boldsymbol{u}_{i}\right), \forall i \\
& \boldsymbol{x}_{t}=\hat{\boldsymbol{x}}_{\text {current }} \\
& \boldsymbol{g}\left(\tau_{t: t+H}\right) \geq \mathbf{0},
\end{array}
$$

where the world model is now a deterministic function $f\left(\boldsymbol{x}_{i}, \boldsymbol{u}_{i}\right)$ and all inequality constraints have been written on standard form as the non-linear vector function $\boldsymbol{g}\left(\tau_{t: t+H}\right) \geq \mathbf{0}$.

Our main contributions for learning safe real-time approximations to the decision problems faced by autonomous robots in uncertain real-world environments instead build on such deterministic planners. These are already mature and established engineering approaches widely used in the robotics and control communities. Both general-purpose and special-purpose planning approaches are available for a range of tasks, ranging from control, to motion and mission planning. For example, there has recently been a surge of interest in fast trajectory solvers for control, both general solvers such as FORCES (Domahidi et al., 2012), ACADO (Houska, Ferreau, and Diehl, 2011) and CasADi (Andersson, Gillis, et al., 2019), and those specialized to certain domains, such as quadcopters (Mellinger and Kumar, 2011).

Here we aim to improve safety and cornputational aspects for planning in real-world uncertain environments by combining such techniques with machine learning approaches. 


\section{Learning to Plan Safe Trajectories under Uncertainty}

As noted in the introduction, ignoring uncertainty like this unfortunately means safety constraints may not hold for real-world autonomous robots, who often have to contend with uncertainty, especially from other agents such as people.

A number of techniques have been proposed in an effort to also make trajectory approaches safe under uncertainty. As we want to satisfy the probabilistic safety constraints from equation (1.3), we here focus on probabilistic approaches. These typically rely on an open-loop approximation, where the constraint uncertainty is propagated over the planning horizon without a feedback policy, either non-parametric particle approximations (Mesbah, 2016) or via Gaussian approximations (Blackmore and Ono, 2009: Vitus and Tomlin, 2011). A trajectory can then be optimized to stay outside the $p \%$ prediction region of any obstacles via regular deterministic constraints. For approximately linear-Gaussian-quadratic problems, an approximate feedback policy can also be included as in LQG above.

Unfortunately, most real-world obstacle avoidance problems are not linear, Gaussian or quadratic, and the open-loop approximations to probabilistic constraints are pessimistic.

In Paper I we examine in greater detail such deterministic approximations of safety constraints for uncertain environments. We focus on obstacle avoidance scenarios with hard-to-predict moving obstacles like people. Such obstacle avoidance is considered a hard problem due to the dynamics and uncertainty involved. Both robot and human dynamics need to be taken into account, and without strong assumptions on prior coordination, the robot has to guarantee safety under possibly unexpected behavior from inattentive humans. Pedestrian obstacle avoidance is a high-risk scenario and such safety constraints need to hold with high probability. We found that existing safety approximations get infeasibly pessimistic in scenarios with moving obstacles. The controller recourse also needs to be included in the planning, e.g. if a pedestrian at some point suddenly turns towards the robot, it has a chance to react to this event and adjust its course. Unfortunately, this would require computing a policy, which is intractable.

We instead propose a novel combination of deterministic trajectory optimization and policy search (c.f. Sec. 3.3. We introduce a parameterization of a soft-constrained MPC program such that it can be seen as a policy $\pi_{\theta}(x)$, and whose constraint parameters $\theta$ can be learned by policy search. By leveraging recent advances in constrained Bayesian optimization (Gelbart, Snoek, and Adams, 2014; Hernández-Lobato et al., 2015), such a policy can automatically be tuned to satisfy probabilistic safety constraints $\operatorname{Pr}\left(\boldsymbol{g}\left(\tau_{t: t+H}\right) \geq \mathbf{0}\right)>p$ with high probability. 
The resulting MPC problem with learned soft constraints via slack variables $\delta$ and safe parameters $\theta^{*}$ then becomes,

$$
\begin{array}{cl}
\underset{\tau_{t: t+H}, \delta}{\arg \min } & c\left(\tau_{t: t+H,} \boldsymbol{\delta}\right) \\
\text { subject to } & \\
& \boldsymbol{x}_{i+1}=\boldsymbol{f}\left(\boldsymbol{x}_{i}, \boldsymbol{u}_{i}\right), \forall i \\
& \boldsymbol{x}_{t}=\hat{\boldsymbol{x}}_{\text {current }} \\
& \boldsymbol{g}_{\theta^{*}}\left(\tau_{t: t+H}\right) \geq \mathbf{0}-\boldsymbol{\delta} \\
& \boldsymbol{\delta} \geq \mathbf{0} .
\end{array}
$$

This is a safe determinized approximation to the original problem with uncertainty, that is fast enough for real-time operation. We demonstrate this in simulation as well as with a real quadcopter. To the best of our knowledge, this is the first real-time approximation to the full control problem under uncertainty, corresponding to equation (1.3), that actually gives calibrated safety constraints for navigating around inattentive moving obstacles.

For longer-range motion planning in complex environments such as offices, the geometric constraints required to not collide with the interior, or its inhabitants, also get similarly complex to satisfy. Here we model the obstacle-free space as $x \in \mathcal{X}_{\text {free. }}$. Optimizing trajectories for these problems may encounter many poor local minima, and just finding a feasible trajectory to the goal is non-trivial. One therefore needs some better method to efficiently find a good global minima for trajectory planning when the robot is constrained to the free-space of such complex environments. This is a computationally challenging problem even without uncertainty. Recent state-ofthe-art trajectory planners first solve a coarse discretized version of the problem and then do local trajectory optimization to refine it (Zhou et al., 2019. Oleynikova et al.,2018).

However, most work on trajectory planning in complex environments only consider static obstacles. In Paper II we extend this to dynamic 3D environments with moving obstacles such as people. The free space in dynamic environments then becomes time-dependent, such that $x \in \mathcal{X}_{\text {free }}\left(t_{i}\right)$. The trajectory optimization problem then becomes,

$$
\begin{array}{cl}
\underset{\tau_{t: t+H}}{\arg \min } & c\left(\tau_{t: t+H}\right) \\
\text { subject to } & \\
& \boldsymbol{x}_{i+1}=\boldsymbol{f}\left(\boldsymbol{x}_{i}, \boldsymbol{u}_{i}\right), \forall i \\
& \boldsymbol{x}_{t}=\hat{\boldsymbol{x}}_{\text {current }} \\
& \boldsymbol{x}_{i} \in \mathcal{X}_{\text {free }}\left(t_{i}\right), \forall i .
\end{array}
$$

We construct a discrete approximation directly to the trajectory optimization problem in (3.6) by generating motion primitives (Frazzoli, Dahleh, and 
Feron, 2002) via optimal control and connecting them into a regular lattice. This makes the problem amenable to graph search algorithms on the lattice approximation. To enable real-time operation we use a multi-resolution temporal lattice that operates in a receding-horizon manner, only planning with regard to dynamic obstacles near the robot.

This is still only a real-time approximation to the deterministic trajectory planning problem. In Paper III we extend this to full motion planning in complex dynamic uncertain environments by generalizing the techniques from Paper I to learn a risk function $\mathcal{R}_{\theta}\left(\tau_{t: t+H}\right)$ representing the risk of a trajectory due to uncertainty. This problem formulation also generalizes the method from learning deterministic safety constraints, to learning risk-aware trajectory planning under arbitrary uncertainty. The trajectory planning problem becomes

$$
\begin{array}{cl}
\underset{\tau_{t: t+H}}{\arg \min } & c\left(\tau_{t: t+H}\right)+\mathcal{R}_{\theta}\left(\tau_{t: t+H}\right) \\
\text { subject to } & \\
& \boldsymbol{x}_{i+1}=\boldsymbol{f}\left(\boldsymbol{x}_{i}, \boldsymbol{u}_{i}\right), \forall i \\
& \boldsymbol{x}_{t}=\hat{\boldsymbol{x}}_{\text {current }} \\
& \boldsymbol{x}_{i} \in \mathcal{X}_{\text {free }}\left(t_{i}\right), \forall i .
\end{array}
$$

We demonstrate this by learning safe motion planners under limited visibility such as obscured corners or doorways, and solve this in real-time with our trajectory planner from Paper II

\section{Learning Neural Network Policies from Trajectory Planning}

While mature and highly optimized trajectory solvers exist for a number of applications, it can still be a challenge to run them in real-time on computationally limited robots. In Paper IV we consider using deep learning to directly improve the computational performance of decision making by learning safe approximations from trajectory planners with safety constraints.

Since the machine learning methods for direct policy optimization that we examined in Section 3.3 still lack robustness in training, in addition to the convergence issues with safety ramifications that we identified in Paper V, we here considered using supervised learning (c.f. Ch. 2) from examples of correct behavior. This is also known as imitation learning (Schaal, 1999) and suffers from a well-known problem in that small imitation errors will accumulate, causing the controller to drift from the distribution of training examples, which results in unpredictable and undesirable behavior. Iterative schemes like DAGGER (Ross, Gordon, and Bagnell, 2011) attempt to at least mitigate this data mismatch problem by testing the learned controller and providing correct examples for its current state distribution. They still have a 
major problem in that all examples are considered equal, but in safety-critical applications some decision are more important than others.

We instead propose a supervised learning transformation that directly addresses problems with safety constraints. We use a risk-aware resampling step to reduce the problem to supervised learning, where the loss function near constraints is amplified to reduce the risk of dangerous constraint violations. This reduces the problem into distinct phases of constrained trajectory optimization and supervised learning. Being agnostic of solvers for either, it allows use of either mature general-purpose solvers, or domain-specific solvers when desired. We use the approximation from Paper I to generate trajectory distributions satisfying probabilistic safety constraints for obstacle avoidance under uncertainty. To efficiently learn deep neural network policies with the large data sets generated by our simulation approach, we use Google's Tensorflow (Abadi et al.,2016) with GPU acceleration.

This approach allows us to reduce the computational cost of the obstacle avoidance controller from Paper I by a factor of 50 to 500, while retaining safety requirements. We demonstrate the approach in a simulated collision avoidance scenario, as well as by implementing it on-board the microcontroller of a $7 \mathrm{~cm}$ nano-quadcopter. To the best of our knowledge, this was the first paper to focus on computational improvements of neural network policies compared to a modern MPC solver on a real robot control application. Competing approaches such as Guided Policy Search (Levine and Koltun, 2013) were, at least at that point, limited to Gaussian trajectory approximations and unconstrained trajectory optimization via iLQG. While we used a simpler approximation, it comes with the advantage of being free to use any trajectory solver with constraints.

\section{Learning Models for Trajectory Planning}

The most obvious way to combine learning with decision-making is to learn better models of reality to reduce uncertainty in the outcome of decisions.

Our chronologically first contribution is from a reinforcement learning perspective on robotics, where the world model is considered entirely unknown. We wanted to leverage recent fast MPC solvers to solve reinforcement learning problems with safety constraints in real-time. In Paper VI we split this problem into learning non-linear models using data efficient and computationally cheap sparse Gaussian processes (c.f. Chapter 2.3), while using the recent fast interior-point QP solver FORCES (Domahidi et al., 2012) via a sequence of convexifications (c.f. sequential quadratic programming, Nocedal and Wright (2006)). To the best of our knowledge, real-time constrained trajectory optimization had not been previously explored by the reinforcement learning community. Handling safety and risk in general has only started to attract interest recently (Garcia and Fernández, 2015), and then usually in the form of risk-averse objectives and discrete MDPs rather 
than the explicit safety constraints in continuous domains that are desirable for robotics. As Gaussian process models are resistant to overfitting even for small data sets, they are unusually data efficient for being highly flexible non-parametric models, which has previously been used to great effect with policy search in PILCO (Deisenroth and Rasmussen, 2011). Although the idea of using trajectory optimization for model-based reinforcement learning was raised some time ago in a sometimes overlooked paper by Atkeson (1998), there has recently been a surge of interest in this area. Two other papers simultaneous to our work also combined Gaussian processes with trajectory optimization (Boedecker et al., 2014: Pan and Theodorou, 2014), but did not consider constraints. Since then there has been an number of further developments, such as using neural network models, that we do not consider here.

While the idea of learning an unknown model is simple in theory, it may not be feasible to guarantee safety on a real robot if there is initially too much uncertainty in the problem. Large uncertainty also causes problems for trajectory approaches who have to rely on approximations for open-loop probabilistic planning. Our optimistic approach in Paper VI is only eventually safe when it has learned the correct world model on a problem with deterministic dynamics. This includes most classical reinforcement learning benchmarks, but not real-world uncertain dynamic environments. This lead us to focus on the risk-adjustment techniques for trajectory planning in Paper I and Paper III.

Finally, in Paper VII we consider learning models for mission planning. We propose to do automatic aerial search for disaster response scenarios. This is a model exploration problem where we have to plan to learn the model as efficiently as possible to minimize harm over time. The model is in this case instead a domain-specific structured spatial model, similar to a Gaussian process with more complex structure. To do real-time probabilistic learning (c.f. Ch 2) in such a model we use INLA (Rue, Martino, and Chopin, 2009), an inference technique tailored to latent Gaussian fields. Since we have to plan to learn the model, this is actually a POMDP where the model parameters are uncertain.

However, we need to plan in real-time, and planning in such POMDPs is extremely intensive even for simpler Gaussian process models (Morere, Marchant, and Ramos, 2017). We instead use a deterministic approximation with a discrete belief-space, where a cell is either known or unknown. This reduces the problem to trajectory planning similar to (3.6), where the state $x_{t}$ is a discretized belief. Similar to how we approximated motion planning in (3.8) with discrete graph search, we approximate this with a Monte-Carlo tree search (Kocsis and Szepesvári, 2006; Browne et al., 2012). Perhaps most famous for its use in AlphaGo (Silver et al., 2017), this is an adaptive tree search algorithm suitable for difficult deterministic sequential decision problems that lack a good heuristic. By preferentially sampling from promis- 
ing branches, the effective branching factor can be significantly reduced. We further tailor it to the problem by using a receding-horizon approach with warm-starts similar to MPC, as well as balancing local exploration against global exploration via a set of long-range macro actions. To the best of our knowledge, this is the first paper to both learn and plan in such a structured disaster response model in real-time.

\section{Conclusions}

In closing, in this chapter we have examined several approaches to solve the sequential decision problem in (1.3) under uncertainty and probabilistic safety constraints. We have considered several combinations of machine learning approaches and optimization-based planning approaches from robotics and control to find safe real-time approximations suitable for autonomous robots navigating dynamic and uncertain environments. We have also considered progressively learning better world models, thereby reducing uncertainty and improving real-time decision-making. 



\section{CHAPTER}

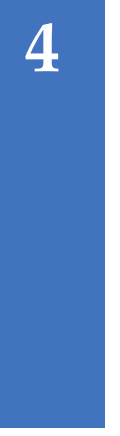

\section{Summary and Discussion}

The aim of this thesis was to examine how recent techniques from machine learning can be used to improve real-time decision-making under uncertainty for autonomous robots. We evaluated several techniques in an attempt to answer two research questions which we reprint from Chapter 1 below.

In the context of autonomous robots:

RQ1 How can robots learn real-time approximations to policy $\pi(\boldsymbol{x})$ in 1.3 that are safe under uncertainty.

RQ2 How can robots learn better internal models $p\left(\boldsymbol{x}_{t+1} \mid \boldsymbol{x}_{t}, \boldsymbol{u}_{t}\right)$ for improved real-time decision making in (1.3).

In this chapter we summarize the included publications before closing with a short discussion of the results and promising avenues for future work.

\subsection{Summary of Contributions}

Paper I Olov Andersson, Mariusz Wzorek, Piotr Rudol, and Patrick Doherty (2016). "Model-Predictive Control with Stochastic Collision Avoidance Using Bayesian Policy Optimization". In: 2016 IEEE International Conference on Robotics and Automation (ICRA).

\section{Video: https://youtu.be/QYYknZ20Zcw}

In Paper I we focus on the difficult problem of dynamically-constrained collision avoidance under uncertainty, with non-cooperative dynamic obstacles such as inattentive pedestrians. We introduce a combination of machine learning and model-predictive control to find a safe 


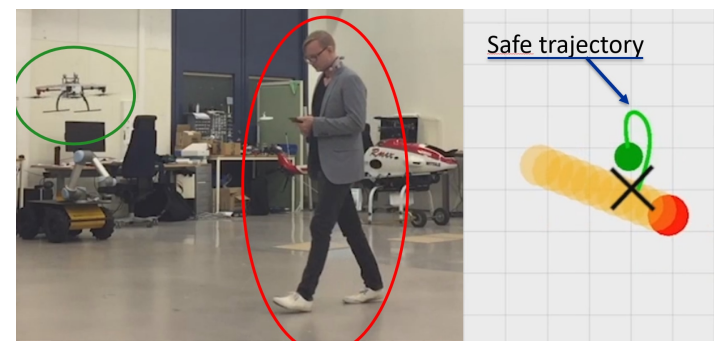

real-time approximation to the decision problem under uncertainty in (1.3). By framing it as a policy search with the policy defined by a model-predictive control solver, we can learn parameterized deterministic soft-constraints that satisfy the original probabilistic constraints $\operatorname{Pr}\left(\boldsymbol{g}\left(\tau_{t: t+H}\right) \geq \mathbf{0}\right)>p$ with the desired confidence $p$. We further propose an effective solution to the policy search problem via constrained Bayesian optimization. We demonstrate the resulting real-time capable MPC approximation in simulation as well as with real quadcopter flights.

Individual contribution: The author of this thesis came up with the idea, wrote most of the software implementation, carried out most of the experiments and wrote most of the paper.

Paper II Olov Andersson*, Oskar Ljungqvist*, Mattias Tiger*, Daniel Axehill, Fredrik Heintz (2018). "Receding-Horizon Lattice-Based Motion Planning with Dynamic Obstacle Avoidance". In: Proceedings of IEEE 57th Annual Conference on Decision and Control (CDC).

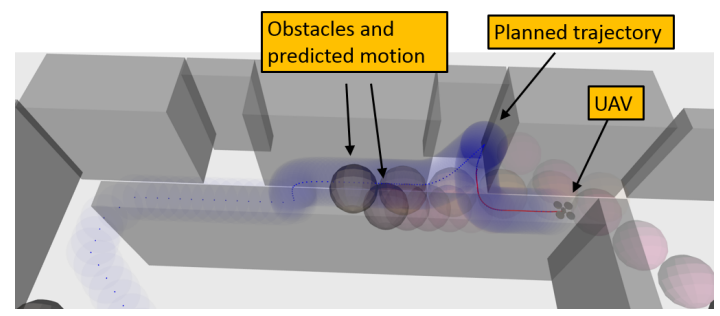

Video: https://youtu.be/1HXJ1zCwQ0I

In Paper II we consider trajectory planning in complex and dynamic 3D environments with moving obstacles such as people. The free space in dynamic environments then becomes time-dependent, a robot may have to plan to wait to let other agents pass. We construct a discrete approximation to the trajectory optimization problem that allows us to pre-compute motion primitives via optimal control and connect them 
into a regular lattice. This makes the problem amenable to graph search algorithms on a temporal lattice approximation. To enable real-time operation we use a multi-resolution lattice that operates in a recedinghorizon manner, only planning with regard to dynamic obstacles near the robot. Trajectory planning is increasingly popular in complex static environments, and this paper extends it to complex dynamic indoor 3D environments, such as offices and warehouses populated by people or other robots. We demonstrate the approach on a simulated DJI Matrice 100 quadcopter in a warehouse environment.

Individual contribution: The author of this thesis along the other two starred authors Oskar Ljungqvist and Mattias Tiger were the main and equal contributors to this publication. This publication sprang out of a project course, where all three authors contributed to all stages of the paper. The most unique contribution from this author was domain expertize from earlier work in quadcopter obstacle avoidance, including modelling and simulation of real quadcopters and their control layers.

Paper III Olov Andersson and Patrick Doherty, "Learning Safe Trajectory Planning in Dynamic Uncertain Environments", Journal article under review.

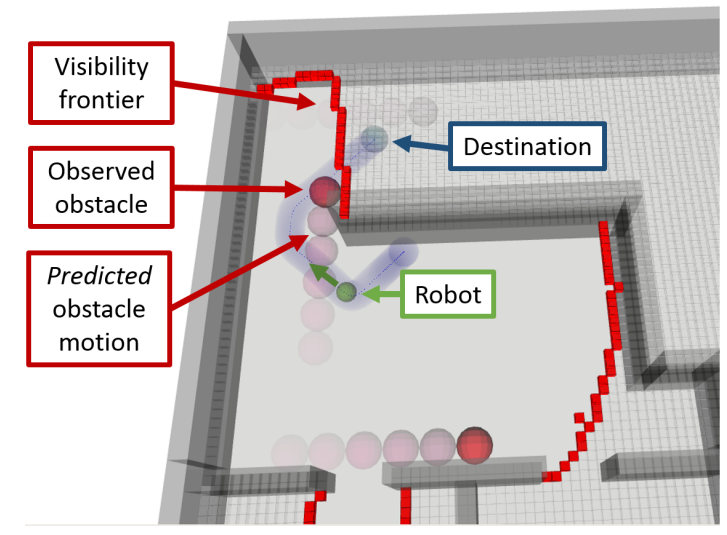

Video: https: / / youtu.be/tndyGFuI7aw

Paper III is a journal article where we generalize Paper I from learning safe deterministic soft-constraints in model-predictive control to learning risk-adjusted trajectory planning under uncertainty in complex, dynamic and uncertain environments. This allows us to handle not only predictive uncertainty from observed pedestrians, but dynamic environments with limited visibility such as occluded corners and doorways. We demonstrate the approach on a simulated indoor environment where it learns to plan safe trajectories around corners and slow 
down when passing through occluded doorways. To the best of our knowledge, this is the first approximation to probabilistic safety constraints in trajectory planning that handles motion uncertainty, limited visibility and results in actual safety levels closely corresponding to the desired ones.

Individual contribution: The author of this thesis came up with the idea, wrote most of the software implementation, carried out most of the experiments and wrote most of the paper.

Paper IV Olov Andersson, Mariusz Wzorek, and Patrick Doherty (2017). “Deep Learning Quadcopter Control via Risk-Aware Active Learning". In: Proceedings of the Thirty-First AAAI Conference on Artificial Intelligence (AAAI).

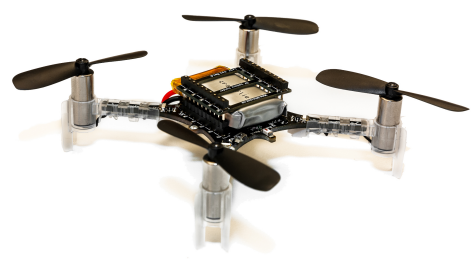

Video: https://youtu.be/xa53w1tyzlo

In Paper IV we evaluate using deep learning with a trajectory solver as teacher to learn fast and safe policy approximations $\hat{\pi}(\boldsymbol{x})$ for real-time use on resource constrained robots. While training a neural network can be computationally intensive, evaluating it is only linear in the number of weights, which is fixed at train time. However, learning a policy approximation via supervised learning has the downside of all examples being treated as equally important, while in real safetycritical applications, it is mainly the behavior in dangerous situations that matters. Deep reinforcement learning approaches take this into account via their objective, but have robustness problems (c.f. Section 3.3. Here we propose a middle ground that generates large data sets of examples from trajectory solvers with safety constraints. It further exploits knowledge of the constraints for risk-aware resampling of data in dangerous regions. This is a heuristic supervised learning approximation that trades some task efficiency for increased safety. However, it is simple to use, agnostic of trajectory solver and allows use of e.g. Google Tensorflow for large data sets. We use the probabilistically safe solver of Paper I and find it is at least 50 times faster on the the collision avoidance domain, while retaining safety. We demonstrate 
this in simulation, as well as by embedding a deep neural network policy on the microcontroller of a $7 \mathrm{~cm}$ nano-quadcopter. This was one of the earliest papers evaluating computational and safety aspects of neural network approximations against a modern trajectory solver in an actual robotics application.

Individual contribution: The author of this thesis came up with the idea and wrote most of the paper. Except for the integration on-board the quadcopter, the author did most of the software implementation and experiments.

Paper V Olov Andersson and Patrick Doherty (2019). “Deep RL for Autonomous Robots: Limitations and Safety Challenges". European Symposium on Neural Networks. An earlier version was presented in the ICML'18 Workshop on Reproducibility in ML.

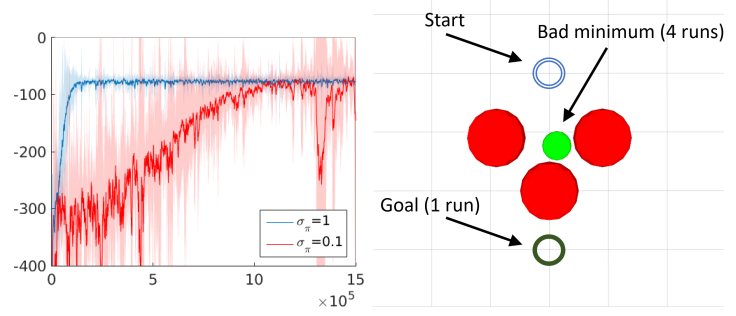

In Paper V we introduce a simplified stochastic obstacle avoidance domain and evaluate a popular policy gradient approach for deep reinforcement learning. There has been a string of papers lately indicating that the standard RL control benchmarks do not contain enough randomization (Rajeswaran et al., 2017; Henderson et al., 2018). We test PPO, which is a leading algorithm on RL control benchmarks (Schulman, Wolski, et al., 2017). We use the simplest possible robot model, but with a penalty for hitting randomly moving obstacles, corresponding to safety constraints. We found convergence very slow, and ultimately failed to find a policy satisfying practical levels of safety. We further found that PPO has surprisingly high variance on such stochastic problems, and local minima can be a significant problem if realistic saturation constraints are included on robot actions. Since these traits are commonly found in the real world, this raises safety concerns for the use of these approaches with autonomous robots.

Individual contribution: The author of this thesis came up with the idea, wrote the software implementation, carried out the experiments and wrote most of the paper.

Paper VI Olov Andersson, Fredrik Heintz, and Patrick Doherty (2015). "ModelBased Reinforcement Learning in Continuous Environments Using 
Real-Time Constrained Optimization". In: Proceedings of the TwentyNinth AAAI Conference on Artificial Intelligence (AAAI).

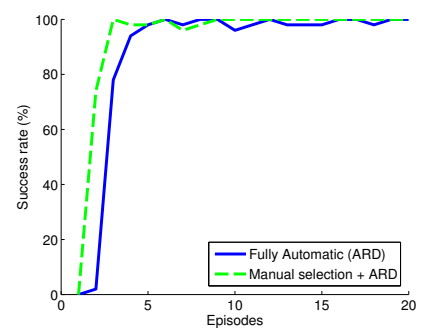

In the chronologically first paper, Paper VI, we consider real-time approximations to the reinforcement learning problem, which assumes unknown world model $p\left(\boldsymbol{x}_{t+1} \mid \boldsymbol{x}_{t}, \boldsymbol{u}_{t}\right)$. We learn this entirely from data via sparse Gaussian processes and propose approximating the policy $\pi(\boldsymbol{x})$ in real-time with recent fast constrained trajectory solvers, also allowing the inclusion of safety constraints $g\left(\tau_{t: t+H}\right) \geq \mathbf{0}$ on the robot. These will be eventually safe if the true model is deterministic and learned to high accuracy. Compared to most global policy approaches, it is computationally tractable to find at least a local minima on the determinized version of the problem. With advances in constrained solvers and computational power, it is increasingly feasible solve these online in real-time. Constrained real-team trajectory optimization, while popular in the control community, does not appear to have been previously considered in the reinforcement learning community. We evaluate it on a simulated RL cart-pole benchmark and a quadcopter domain.

Individual contribution: The author of this thesis came up with the idea, wrote the software implementation, carried out the experiments and wrote nearly all of the paper.

Paper VII Olov Andersson*, Per Sidén*, Johan Dahlin, Patrick Doherty, Mattias Villani (2019). "Real-Time Robotic Search using Structural Spatial Point Processes". In: Proceedings of the Thirty-Fifth Conference on Uncertainty in Artificial Intelligence (UAI).

\section{Video: https://youtu.be/okbAfeGIYbI}

In Paper VII we consider real-time learning and search planning for automatic aerial victim search in disaster response. The proposed solution attempts to capture all problem desiderata in a structured probabilistic model, including population density, the probability of injury, and chance of detection via UAV. It allows informative priors from e.g. geographic information systems or cell-phone traffic data to be 


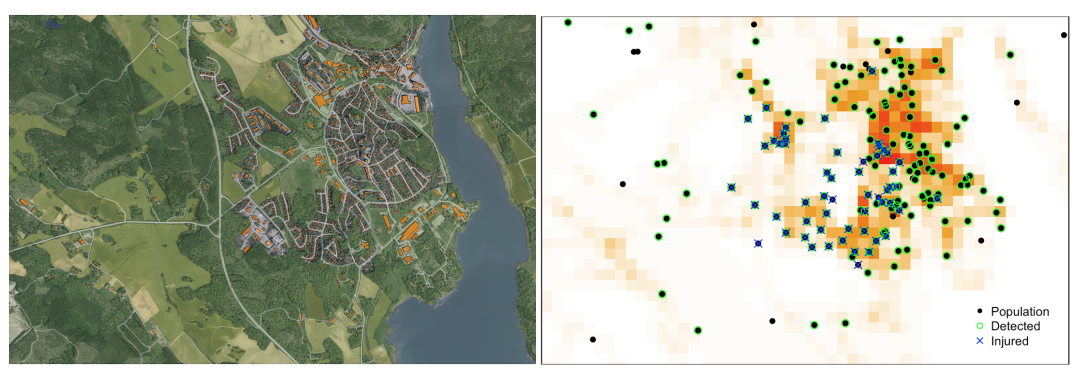

included, but it can also learn these individually via spatial point processes. Both probabilistic learning and search planning are computationally hard problems. For real-time learning we use an integrated nested-Laplace approximation tailored to such latent Gaussian fields. The search problem is a POMDP that directly minimizes victim harm. We use a deterministic belief-space approximation via a novel variant of Monte-Carlo tree-search tailored to the problem. This includes receding-horizon operation, warm-starts, and long-range macro actions. Instead of a separate task objective with constraints on safety, safety is the objective of this task.

Individual contribution: The author of this thesis along with Per Sidén were main and equal contributors to this publication. In particular, the author suggested the structure with injury field and GIS-based prior, introduced the harm-based optimization objective, as well as the tailored Monte-Carlo tree search to minimize it. The implementation and experiments were mostly done by the author and Per Sidén. 


\subsection{Discussion and Future Work}

We have found novel approximations to the decision problem in equation (1.3) that enabled us to learn safe planning and control for computationally constrained robotic platforms in dynamic uncertain environments. This was demonstrated by going from simulation, to mid-sized quadcopter, down to onboard implementation on a nano-quadcopter. A theme throughout this research has been a focus on close integration and evaluation with real robotic systems such as the DJI Matrice 100, Bitcraze Crazyflie and LinkQuad quadcopter platforms. However, none of the proposed methods are limited to quadcopters. Although domain-specific assumptions, about the world transition model $p\left(\boldsymbol{x}_{t+1} \mid \boldsymbol{x}_{t}, \boldsymbol{u}_{t}\right)$ or in the type of trajectory optimization used to approximate (1.3), can improve their computational performance.

As shown in Figure 1.3 the research in this thesis can be organized into three different threads pertaining to learning approaches for autonomous robots in dynamic and uncertain environments (DUE):

i) Learning risk-adjustments for trajectory planning in DUE

ii) Learning neural-network decision policies for DUE

iii) Learning world models in real-time to reduce uncertainty in DUE

The first two threads address RQ1, the first research question in Chapter 1 . Taken together, the contributions in Paper I to Paper V at least provide promising avenues for learning safe real-time approximations to decision making under uncertainty in autonomous robots.

The third thread addresses RQ2 - how can autonomous robots learn better models for improved real-time decision making. We provide some examples using probabilistic machine learning with Paper VI and Paper VII, but we have so far only scratched the surface of this very broad question.

All three threads seem worthwhile to pursue further, and we attempt to give a brief outline below. However, all our experiments with real robots have generated a wealth of new research questions, more than can feasibly be covered here.

In brief, the first thread on learning risk-adjustments for trajectory planners can be extended in multiple ways, as already outlined in Paper III. With better parallelization and some further work on calibrating conservative simulation models against real-world safety levels, we believe it might be ready for wider use, aiding the development of future autonomous robots.

The second thread focused on learning neural network policies, which is a very active research area at the moment. These hold great promise to automate the feature construction required in our first research thread, while potentially getting computational benefits as shown in Paper IV. However, these policy optimization approaches still have considerable robustness concerns that have to be remedied for use with autonomous robots. The insights 
gleaned while experimenting with Paper V could likely be leveraged into improving their safety and robustness.

The third and final research thread in turn attempted to address how to progressively learn better world models for the real-time decision-making of autonomous robots. While pre-trained models can be used in a number of ways, the currently most mature approach for safely learning models autonomously in real robots seem to be probabilistic models tailored to domain structure such as in Paper VII.

The tabula rasa assumption common in reinforcement learning is seemingly simply too dangerous to use with many real-world robots. Learning structured and semi-parametric models, where only carefully selected parts of the environment may be entirely unknown, seems more promising. Indeed, many conventional robotics problems such as simultaneous localization and mapping (Thrun, Burgard, and Fox, 2005b) can be seen as special cases of this.

While neural networks have shown promise in also learning the structure in some domains like vision, autonomously learning neural networks in realtime still presents a number of difficulties. One is that they are prone to overfitting without somebody to tune the training. A second problem is that it is difficult to quantify their uncertainty for decision making - what they actually know. Finally, a third problem is how to robustly combine such neural network learning with conventional parametric models and domain insight when such is available. 



\section{Bibliography}

Abadi, Martin, Paul Barham, Jianmin Chen, Zhifeng Chen, Andy Davis, Jeffrey Dean, Matthieu Devin, Sanjay Ghemawat, Geoffrey Irving, Michael Isard, et al. (2016). “TensorFlow: A system for large-scale machine learning". In: Proceedings of the 12th USENIX Symposium on Operating Systems Design and Implementation (OSDI). Savannah, Georgia, USA.

Abbeel, Pieter, Adam Coates, and Andrew Y Ng (2010). "Autonomous helicopter aerobatics through apprenticeship learning". In: The International Journal of Robotics Research 29.13, pp. 1608-1639.

Andersson, Joel A E, Joris Gillis, Greg Horn, James B Rawlings, and Moritz Diehl (2019). "CasADi - A software framework for nonlinear optimization and optimal control". In: Mathematical Programming Computation 11.1, pp. 1-36. DOI: $10.1007 / \mathrm{s} 12532-018-0139-4$.

Andersson, Olov and Patrick Doherty (2019). "Deep RL for Autonomous Robots: Limitations and Safety Challenges". In: Proceedings of the 27th European Symposium on Neural Networks. An earlier version was presented at ICML'18 Workshop on Reproducible ML. ESANN, pp. 489-495.

- (2020). "Learning Safe Trajectory Planning in Dynamic Uncertain Environments". Journal article under review.

Andersson, Olov, Fredrik Heintz, and Patrick Doherty (2015). "Model-Based Reinforcement Learning in Continuous Environments Using Real-Time Constrained Optimization". In: Proceedings of the Twenty-Ninth AAAI Conference on Artificial Intelligence ( $A A A I)$. Ed. by Blai Bonet and Sven Koenig. AAAI Press, pp. 2497-2503. ISBN: 978-1-57735-698-1.

Andersson, Olov, Oskar Ljungqvist, Mattias Tiger, Daniel Axehill, and Fredrik Heintz (2018). "Receding-horizon lattice-based motion planning with dynamic obstacle avoidance". In: 2018 IEEE Conference on Decision and Control (CDC). IEEE, pp. 4467-4474. 
Andersson, Olov, Per Sidén, Johan Dahlin, Patrick Doherty, and Mattias Villani (2019). "Real-Time Robotic Search using Structural Spatial Point Processes". In: Proceedings of the Thirty-Fifth Conference on Uncertainty in Artificial Intelligence, UAI 2019, Tel Aviv, Israel, July 22-25, 2019. AUAI Press.

Andersson, Olov, Mariusz Wzorek, and Patrick Doherty (2017). “Deep Learning Quadcopter Control via Risk-Aware Active Learning". In: Proceedings of the Thirty-First AAAI Conference on Artificial Intelligence (AAAI). San Francisco, USA, February 4-9: AAAI Press, pp. 3812-3818.

Andersson, Olov, Mariusz Wzorek, Piotr Rudol, and Patrick Doherty (May 2016). "Model-Predictive Control With Stochastic Collision Avoidance Using Bayesian Policy Optimization". In: 2016 IEEE International Conference on Robotics and Automation (ICRA), pp. 4597-4604. DOI: $10.1109 /$ ICRA.2016.7487661.

Arntz M., T. Gregory and U. Zierahn (2016). "The Risk of Automation for Jobs in OECD Countries". In: DOI: http : / / dx . doi . org / 10 . 1787 / 5jlz9h56dvq7 - en. URL: / content/workingpaper / $5 j l z 9 h 56 d v q 7-e n$.

Åstrom, Karl Johan (1970). Introduction to stochastic control theory. eng. Vol. 70. Mathematics in science and engineering. Academic Press. ISBN: 0-12065650-7.

Atkeson, Christopher G (1998). “Nonparametric model-based reinforcement learning". In: Advances in neural information processing systems 10. Morgan Kaufmann Publishers, pp. 1008-1014.

Bertsekas, Dimitri P (2011). “Dynamic programming and optimal control 3rd edition, volume II". In: Belmont, MA: Athena Scientific.

- (2005). Dynamic Programming and Optimal Control, Vol. I. 3rd. Athena Scientific.

- (2012). Dynamic Programming and Optimal Control, Vol. II. 4rd. Athena Scientific.

Bishop, Christopher M (2006). Pattern recognition and machine learning. Springer.

Blackmore, Lars and Masahiro Ono (2009). "Convex chance constrained predictive control without sampling". In: Proceedings of the AIAA Guidance, Navigation and Control Conference, pp. 7-21.

Boedecker, Joschka, Jost Tobias Springenberg, Jan Wülfing, and Martin Riedmiller (2014). "Approximate real-time optimal control based on sparse gaussian process models". In: Adaptive Dynamic Programming and Reinforcement Learning (ADPRL), 2014 IEEE Symposium on. IEEE, pp. 1-8.

Boston Dynamics (2019). Spot. https : / / www . bostondynamics . com / spot. [Online; accessed 16-Dec-2019].

Browne, Cameron B, Edward Powley, Daniel Whitehouse, Simon M Lucas, Peter I Cowling, Philipp Rohlfshagen, Stephen Tavener, Diego Perez, Spyridon Samothrakis, and Simon Colton (2012). "A survey of Monte 
Carlo tree search methods". In: IEEE Transactions on Computational Intelligence and AI in games 4.1, pp. 1-43.

Deisenroth, Marc Peter, Gerhard Neumann, Jan Peters, et al. (2013). "A Survey on Policy Search for Robotics." In: Foundations and Trends in Robotics 2.1-2, pp. 1-142.

Deisenroth, Marc and Carl Edward Rasmussen (2011). "PILCO: A modelbased and data-efficient approach to policy search". In: Proceedings of the 28th International Conference on Machine Learning (ICML-11), pp. 465-472.

Domahidi, A., A. Zgraggen, M.N. Zeilinger, M. Morari, and C.N. Jones (Dec. 2012). "Efficient interior point Methods for Multistage Problems Arising in Receding Horizon Control". In: IEEE Conference on Decision and Control (CDC). Maui, HI, USA, pp. 668-674.

Duan, Yan, Xi Chen, Rein Houthooft, John Schulman, and Pieter Abbeel (2016). "Benchmarking deep reinforcement learning for continuous control". In: Proceedings of the 33rd International Conference on Machine Learning (ICML).

Frazzoli, Emilio, Munther A Dahleh, and Eric Feron (2002). "Real-time motion planning for agile autonomous vehicles". In: Journal of guidance, control, and dynamics 25.1, pp. 116-129.

Frey, Carl Benedikt and Michael A Osborne (2017). "The future of employment: how susceptible are jobs to computerisation?" In: Technological Forecasting and Social Change 114, pp. 254-280.

Gal, Yarin, Rowan McAllister, and Carl Edward Rasmussen (2016). "Improving PILCO with Bayesian neural network dynamics models". In: DataEfficient Machine Learning workshop, ICML. Vol. 4, p. 34.

Garcia, Javier and Fernando Fernández (2015). "A comprehensive survey on safe reinforcement learning". In: Journal of Machine Learning Research 16.1, pp. 1437-1480.

Gelbart, Michael, Jasper Snoek, and Ryan Adams (2014). "Bayesian Optimization with Unknown Constraints". In: Proceedings of the Thirtieth Conference Annual Conference on Uncertainty in Artificial Intelligence (UAI-14). Corvallis, Oregon: AUAI Press, pp. 250-259.

Gelman, A., J.B. Carlin, H.S. Stern, and D.B. Rubin (2003). Bayesian Data Analysis, Second Edition. Taylor \& Francis. ISBN: 9781584883883.

Georgiou, T. T. and A. Lindquist (Oct. 2013). "The Separation Principle in Stochastic Control, Redux". In: IEEE Transactions on Automatic Control 58.10, pp. 2481-2494. ISSN: 2334-3303. DOI: 10 . 1109/ TAC . 2013 . 2259207.

Goodfellow, Ian, Yoshua Bengio, and Aaron Courville (2016). Deep Learning. http://www.deeplearningbook.org. MIT Press.

Henderson, Peter, Riashat Islam, Philip Bachman, Joelle Pineau, Doina Precup, and David Meger (2018). "Deep reinforcement learning that matters". In: Thirty-Second AAAI Conference on Artificial Intelligence. 
Hernández-Lobato, José Miguel, Michael A. Gelbart, Matthew W. Hoffman, Ryan P. Adams, and Zoubin Ghahramani (2015). "Predictive Entropy Search for Bayesian Optimization with Unknown Constraints". In: Proceedings of the 32nd International Conference on Machine Learning (ICML), pp. 1699-1707.

Houska, B., H.J. Ferreau, and M. Diehl (2011). "ACADO Toolkit - An Open Source Framework for Automatic Control and Dynamic Optimization". In: Optimal Control Applications and Methods 32.3, pp. 298-312.

Kakade, Sham M (2002). "A Natural Policy Gradient". In: Advances in Neural Information Processing Systems 14. Ed. by T. G. Dietterich, S. Becker, and Z. Ghahramani. MIT Press, pp. 1531-1538.

Kingma, Diederik and Jimmy Ba (2015). "Adam: A method for stochastic optimization". In: International Conference on Learning Representations (ICLR 2015), San Diego, 2015.

Knight, Frank H. (1921). Risk, uncertainty and profit, Boston; New York: Houghton Mifflin Co. URL: http: / / books . google.com / books? id=BzU6AAAAMAAJ.

Kober, Jens and Jan Peters (2012). "Reinforcement learning in robotics: A survey". In: Reinforcement Learning. Springer, pp. 579-610.

Kocsis, Levente and Csaba Szepesvári (2006). "Bandit based Monte-Carlo planning". In: European conference on machine learning. Springer, pp. 282293.

Koller, Daphne and Nir Friedman (2009). Probabilistic graphical models: principles and techniques. MIT press.

Levine, Sergey and Vladlen Koltun (2013). "Guided Policy Search." In: ICML (3), pp. 1-9.

Lillicrap, Timothy P, Jonathan J Hunt, Alexander Pritzel, Nicolas Heess, Tom Erez, Yuval Tassa, David Silver, and Daan Wierstra (2015). "Continuous control with deep reinforcement learning". In: arXiv preprint arXiv:1509.02971.

Mania, Horia, Aurelia Guy, and Benjamin Recht (2018). "Simple random search of static linear policies is competitive for reinforcement learning". In: Advances in Neural Information Processing Systems 31. Ed. by S. Bengio, H. Wallach, H. Larochelle, K. Grauman, N. Cesa-Bianchi, and R. Garnett. Curran Associates, Inc., pp. 1800-1809.

Mayne, David (1966). "A Second-order Gradient Method for Determining Optimal Trajectories of Non-linear Discrete-time Systems". In: International Journal of Control 3.1, pp. 85-95. DOI: 10 . 1080/ 00207176608921369 .

Mellinger, Daniel and Vijay Kumar (2011). "Minimum snap trajectory generation and control for quadrotors". In: 2011 IEEE International Conference on Robotics and Automation. IEEE, pp. 2520-2525. 
Mesbah, A. (Dec. 2016). "Stochastic Model Predictive Control: An Overview and Perspectives for Future Research". In: IEEE Control Systems Magazine 36.6, pp. 30-44. ISSN: 1941-000X. DOI: $10.1109 /$ MCS .2016.2602087.

Mnih, Volodymyr, Koray Kavukcuoglu, David Silver, Andrei A Rusu, Joel Veness, Marc G Bellemare, Alex Graves, Martin Riedmiller, Andreas K Fidjeland, Georg Ostrovski, et al. (2015). "Human-level control through deep reinforcement learning". In: Nature 518.7540, pp. 529-533.

Morere, Philippe, Roman Marchant, and Fabio Ramos (2017). "Sequential Bayesian optimization as a POMDP for environment monitoring with UAVs". In: 2017 IEEE International Conference on Robotics and Automation (ICRA). IEEE, pp. 6381-6388.

Nocedal, J. and S. J. Wright (2006). Numerical Optimization. 2nd. New York: Springer.

NTSB, National Transportation Safety Board (2019). Vehicle Automation Report, Tempe, AZ, HWY18MH010. URL: https : / / dms . ntsb - gov / pubdms / search / document . cfm ? docID $=477717 \&$ docket ID $=$ $62978 \& \mathrm{mkey}=96894$.

Oleynikova, Helen, Zachary Taylor, Roland Siegwart, and Juan Nieto (2018). "Sparse 3d topological graphs for micro-aerial vehicle planning". In: 2018 IEEE/RSJ International Conference on Intelligent Robots and Systems (IROS). IEEE, pp. 1-9.

Pan, Yunpeng and Evangelos Theodorou (2014). "Probabilistic Differential Dynamic Programming". In: Advances in Neural Information Processing Systems 27. Ed. by Z. Ghahramani, M. Welling, C. Cortes, N. D. Lawrence, and K. Q. Weinberger. Curran Associates, Inc., pp. 1907-1915.

Parmas, Paavo, Carl Edward Rasmussen, Jan Peters, and Kenji Doya (2019). "PIPPS: Flexible model-based policy search robust to the curse of chaos". In: arXiv preprint arXiv:1902.01240.

Powell, Warren B (2007). Approximate Dynamic Programming: Solving the curses of dimensionality. Vol. 703. John Wiley \& Sons.

Rajeswaran, Aravind, Kendall Lowrey, Emanuel V Todorov, and Sham M Kakade (2017). "Towards generalization and simplicity in continuous control". In: Advances in Neural Information Processing Systems, pp. 65506561.

Rasmussen, Carl and Christopher K.I. Williams (2006). Gaussian Processes for Machine Learning. Cambridge, MA: MIT Press.

Ross, Stéphane, Geoffrey J. Gordon, and Drew Bagnell (2011). “A Reduction of Imitation Learning and Structured Prediction to No-Regret Online Learning". In: Proceedings of the Fourteenth International Conference on Artificial Intelligence and Statistics, AISTATS 2011, Fort Lauderdale, USA, April 11-13, 2011, pp. 627-635.

Rue, Håvard, Sara Martino, and Nicolas Chopin (2009). “Approximate Bayesian inference for latent Gaussian models by using integrated nested 
Laplace approximations". In: Journal of the Royal Statistical Society: Series B 71.2, pp. 319-392.

Schaal, S. (1999). "Is imitation learning the route to humanoid robots?" In: Trends in cognitive sciences 3.6, pp. 233-242.

Schulman, John, Sergey Levine, Pieter Abbeel, Michael I Jordan, and Philipp Moritz (2015). “Trust Region Policy Optimization." In: Proceedings of The 32nd International Conference on Machine Learning. Ed. by Francis Bach and David Blei, pp. 1889-1897.

Schulman, John, Filip Wolski, Prafulla Dhariwal, Alec Radford, and Oleg Klimov (2017). "Proximal policy optimization algorithms". In: arXiv preprint arXiv:1707.06347.

Silver, David, Julian Schrittwieser, Karen Simonyan, Ioannis Antonoglou, Aja Huang, Arthur Guez, Thomas Hubert, Lucas Baker, Matthew Lai, Adrian Bolton, et al. (2017). "Mastering the game of go without human knowledge". In: Nature 550.7676, p. 354.

Simon, Herbert A (1955). "A behavioral model of rational choice". In: The quarterly journal of economics 69.1, pp. 99-118.

Snelson, Edward and Zoubin Ghahramani (2006). "Sparse Gaussian Processes using Pseudo-inputs". In: Advances in Neural Information Processing Systems 18. Ed. by Y. Weiss, B. Schölkopf, and J. Platt. Cambridge, MA: MIT Press, pp. 1257-1264.

Srivastava, Nitish, Geoffrey Hinton, Alex Krizhevsky, Ilya Sutskever, and Ruslan Salakhutdinov (2014). "Dropout: A simple way to prevent neural networks from overfitting". In: The Journal of Machine Learning Research 15.1, pp. 1929-1958.

Sutton, Richard S. and Andrew G. Barto (1998). Introduction to Reinforcement Learning. 1st. Cambridge, MA, USA: MIT Press. ISBN: 0262193981.

- (2018). Introduction to Reinforcement Learning. 2nd. Cambridge, MA, USA: MIT Press. ISBN: 9780262039246.

Tassa, Yuval, Tom Erez, and Emanuel Todorov (2012). "Synthesis and stabilization of complex behaviors through online trajectory optimization". In: 2012 IEEE/RSJ International Conference on Intelligent Robots and Systems. IEEE, pp. 4906-4913.

Thrun, Sebastian, Wolfram Burgard, and Dieter Fox (2005a). Probabilistic Robotics. MIT Press.

- (2005b). Probabilistic robotics. MIT press.

Todorov, E. and Weiwei Li (2004). "Iterative linear-quadratic regulator design for nonlinear biological movement systems". In: First International Conference on Informatics in Control, Automation and Robotics. N.P.: INSTICC Press., 222-229 vol. 1.

Udacity (2016). Udacity Self-Driving Car Driving Data 10/3/2016 (dataset-22.bag.tar.gz). URL: https://github.com/udacity/self-drivingcar 
Vitus, Michael P. and C.J. Tomlin (May 2011). “Closed-loop belief space planning for linear, Gaussian systems". In: 2011 IEEE International Conference on Robotics and Automation (ICRA), pp. 2152-2159. DOI:10.1109/ICRA. 2011.5980257

Watkins, Christopher JCH and Peter Dayan (1992). "Q-learning”. In: Machine learning 8.3-4, pp. 279-292.

Williams, Ronald J (1992). "Simple statistical gradient-following algorithms for connectionist reinforcement learning". In: Machine learning 8.3-4, pp. 229-256.

Zhou, Boyu, Fei Gao, Luqi Wang, Chuhao Liu, and Shaojie Shen (2019). "Robust and efficient quadrotor trajectory generation for fast autonomous flight". In: IEEE Robotics and Automation Letters 4.4, pp. 3529-3536. 


\section{Papers}

The papers associated with this thesis have been removed for copyright reasons. For more details about these see:

http://urn.kb.se/resolve?urn=urn:nbn:se:liu:diva-163419 


\section{FACULTY OF SCIENCE AND ENGINEERING}

Linköping Studies in Science and Technology, Dissertation No. 2051, 2020

Department of Computer and Information Science

Linköping University

SE-581 83 Linköping, Sweden

wWw.liu.se 